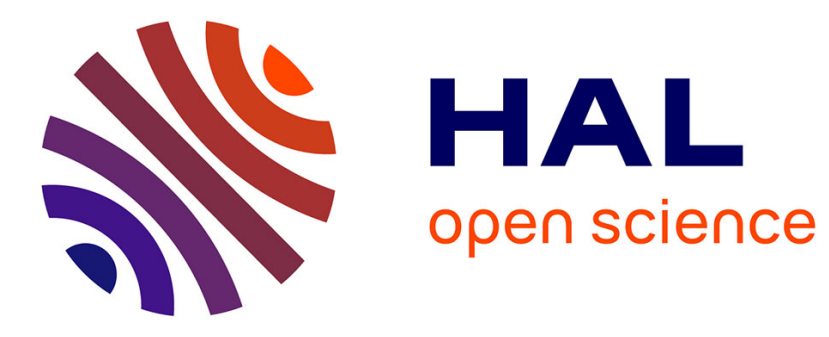

\title{
Solar flares at submillimeter wavelengths
}

Säm Krucker, C. G. Giménez de Castro, H. S. Hudson, G. Trottet, T. S. Bastian, A. S. Hales, J. Kašparová, K.-L. Klein, Matthieu Kretzschmar, T. Lüthi, et al.

\section{- To cite this version:}

Säm Krucker, C. G. Giménez de Castro, H. S. Hudson, G. Trottet, T. S. Bastian, et al.. Solar flares at submillimeter wavelengths. Astronomy and Astrophysics Review, 2013, 21 (1), pp.58. 10.1007/s00159-013-0058-3 . insu-01256245

\section{HAL Id: insu-01256245 https://hal-insu.archives-ouvertes.fr/insu-01256245}

Submitted on 15 May 2020

HAL is a multi-disciplinary open access archive for the deposit and dissemination of scientific research documents, whether they are published or not. The documents may come from teaching and research institutions in France or abroad, or from public or private research centers.
L'archive ouverte pluridisciplinaire HAL, est destinée au dépôt et à la diffusion de documents scientifiques de niveau recherche, publiés ou non, émanant des établissements d'enseignement et de recherche français ou étrangers, des laboratoires publics ou privés. 


\title{
Solar flares at submillimeter wavelengths
}

\author{
Säm Krucker • C.G. Giménez de Castro • H.S. Hudson • G. Trottet • \\ T.S. Bastian • A.S. Hales • J. Kašparová • K.-L. Klein • M. Kretzschmar • \\ T. Lüthi · A. Mackinnon · S. Pohjolainen · S.M. White
}

Received: 17 November 2011 / Published online: 23 March 2013

(C) Springer-Verlag Berlin Heidelberg 2013

\begin{abstract}
We discuss the implications of the first systematic observations of solar flares at submillimeter wavelengths, defined here as observing wavelengths shorter than $3 \mathrm{~mm}$ (frequencies higher than $0.1 \mathrm{THz}$ ). The events observed thus far show that this wave band requires a new understanding of high-energy processes in solar flares. Several events, including observations from two different observatories, show
\end{abstract}

\author{
S. Krucker $(\bowtie)$ \\ Institute of 4D Technologies, School of Engineering, University of Applied Sciences and Arts \\ Northwestern Switzerland, 5210 Windisch, Switzerland \\ e-mail: krucker@ssl.berkeley.edu \\ C.G. Giménez de Castro \\ CRAAM, Universidade Presbiteriana Mackenzie, São Paulo, SP 01302-907, Brazil \\ S. Krucker · H.S. Hudson \\ Space Sciences Laboratory, University of California, Berkeley, CA 94720-7450, USA \\ G. Trottet · K.-L. Klein \\ Observatoire de Paris, LESIA, CNRS-UMR 8109, Meudon, France
}

T.S. Bastian · A.S. Hales

National Radio Astronomy Observatory, Charlottesville, VA 22903, USA

J. Kašparová

Astronomical Institute of the Academy of Sciences of the Czech Republic, 25165 Ondřejov, Czech Republic

M. Kretzschmar

LPC2E, UMR 6115 CNRS et Université d'Orléans, Orléans Cedex 2, France

T. Lüthi

Leica Geosystem AG, Hexagon Metrology, Moenchmattweg 5, 5035 Unterentfelden, Switzerland

A. Mackinnon

DACE/Department of Physics and Astronomy, University of Glasgow, Glasgow, UK 
during the impulsive phase of the flare a spectral component with a positive (increasing) slope at the highest observable frequencies (up to $405 \mathrm{GHz}$ ). To emphasize the increasing spectra and the possibility that these events could be even more prominent in the THz range, we term this spectral feature a "THz component". Here we review the data and methods, and critically assess the observational evidence for such distinct component(s). This evidence is convincing. We also review the several proposed explanations for these feature(s), which have been reported in three distinct flare phases. These data contain important clues to flare development and particle acceleration as a whole, but many of the theoretical issues remain open. We generally have lacked systematic observations in the millimeter-wave to far-infrared range that are needed to complete our picture of these events, and encourage observations with new facilities.

Keywords Sun: flares $\cdot$ Sun: radio observations

\title{
1 Introduction
}

During a solar flare the Sun can become very bright at microwave frequencies, with fluxes at the Earth exceeding $10^{5} \mathrm{SFU}^{1}$ in the $1-100 \mathrm{GHz}$ band. The emission mechanism for such a microwave burst is well explained by incoherent gyrosynchrotron emission from electrons with a power-law energy distribution moving through magnetic fields of 100-1000 G above active regions (e.g. Bastian et al. 1998). Typically these electrons radiate at moderate harmonics of the electron cyclotron frequency $v_{c} \approx 2.8 B \mathrm{MHz}$, where $B$ is the field strength in $\mathrm{G}$, with fluxes declining above a spectral peak in the harmonic range 5-10. Generally the higher frequencies reflect the presence of higher-energy electrons, with the peak emission frequency scaling as $\gamma^{2}$ in the relativistic limit. As one moves to higher frequencies above the microwave range, the falling flux of gyrosynchrotron radiation and other flare emissions must compete with the rising flux of the solar disk, which increases with frequency at roughly the Rayleigh-Jeans rate $\left(f_{v} \propto v^{2}\right)$. Accordingly, the gyrosynchrotron emission from flares becomes harder to detect at the higher frequencies.

The striking discovery that motivates the present review lies in the spectrum observed above $100 \mathrm{GHz}$, where some events appear to exhibit an unexpected upturn towards the THz domain (Kaufmann et al. 2004). The events having mm-wave spectra with positive slopes (the $\mathrm{THz}$ events), may have both impulsive and gradual components, but the positive-slope spectra have been well observed thus far in only a handful of the most energetic events (Table 1).

\footnotetext{
${ }^{1}$ One solar flux unit $(\mathrm{SFU})=10^{-19} \mathrm{ergs} \mathrm{cm}^{-2} \mathrm{~s}^{-1} \mathrm{~Hz}^{-1}=10^{-22} \mathrm{~W} \mathrm{~m}^{-2} \mathrm{~Hz}^{-1}$.
}

\author{
S. Pohjolainen \\ Department of Physics and Astronomy, Tuorla Observatory, University of Turku, 21500 Piikkiö, \\ Finland
}


We discuss observations of the "normal" gyrosynchrotron spectrum for reference in Sect. 3.1, and the THz events in Sect. 3.2. Section 3.3 addresses the credibility of these "THz event" observations directly. Surprisingly diverse theoretical ideas for the $\mathrm{THz}$ events have already appeared-our discussion in Sect. 4 lists ten distinct mechanisms (see Table 2). The basic free-free and gyrosynchrotron emission mechanisms are forced to extreme parameter ranges by the $\mathrm{THz}$ events, and several more exotic and less-well-defined ideas have appeared. This review systematically covers instrumentation, observations, and interpretation. A reader uninterested in full details can get an idea of the observations from Table 1, Fig. 7, and Sect. 3.3, and of the interpretations from Table 2 .

\section{Instrumentation}

\subsection{Background}

Early observations showed that solar flares could produce powerful mm-wave radio bursts. In spite of various observational difficulties (small dishes, poor atmospheric conditions, simple radiometers etc.), there were several successful results. The flare SOL1969-03-27T13:26 $6^{2}$ was detected both by Croom (1970) at $71 \mathrm{GHz}$, with a flux density of $4.8 \times 10^{3} \mathrm{SFU}$, and by Cogdell (1972) at $95 \mathrm{GHz}$ at a comparable flux density. The SOL1991-06-04 and SOL1991-06-06 events produced $80 \mathrm{GHz}$ fluxes of $1.4 \times 10^{5}$ and $1.6 \times 10^{5} \mathrm{SFU}$, respectively (in both of these events the $80 \mathrm{GHz}$ flux was higher than the $35 \mathrm{GHz}$ flux, so the peak in the radio spectrum appeared to be above 35 GHz; Ramaty et al. 1994).

In these cases the usual microwave event seemed to extend to unusually high frequencies, with no indication that the mm-wave emission might be distinct from the common cm wave burst. The events reported by Akabane et al. (1973) and Kaufmann et al. (1985) were different. In these cases the flux density was significantly higher at $73 \mathrm{GHz}$ and $90 \mathrm{GHz}$, respectively, than at lower frequencies. Akabane et al. interpreted their event as thermal in nature, suggesting a source "close to the H $\alpha$ region." The multiple peaks of the Kaufmann et al. event, which was not even detectable at $7 \mathrm{GHz}$, suggested a non-thermal origin for the high-frequency component. The flux density profile at $90 \mathrm{GHz}$ resembled the count rate time profile of hard X-rays in the 24-108 keV range, while the microwaves at lower frequencies did not. This is unusual, since burst profiles at $\mathrm{cm}$ wavelengths often resemble the hard X-ray profiles (e.g. Kosugi et al. 1988). The brightest peaks at $90 \mathrm{GHz}$ occurred in the early phase of the event, and were hardly discernible at $30 \mathrm{GHz}$. This event hence seems to be the first, and possibly still the only, example of a solar burst whose microwave emission is restricted to $\mathrm{mm}$ (and possibly shorter) waves. The peculiarities of this event and its relationship with the hard X-rays inspired a wide variety of interpretations, including thermal and non-thermal gyrosynchrotron radiation and synchrotron/inverse Compton processes (McClements and Brown 1986; De Jager et al. 1987; Klein 1987).

\footnotetext{
${ }^{2}$ We adopt the IAU target identification scheme here: any solar event can be specified as SOLyyyy-mmdd:Thh:mm:ss in time, and position coordinates can also be added for disambiguation if needed (Leibacher et al. 2010).
} 

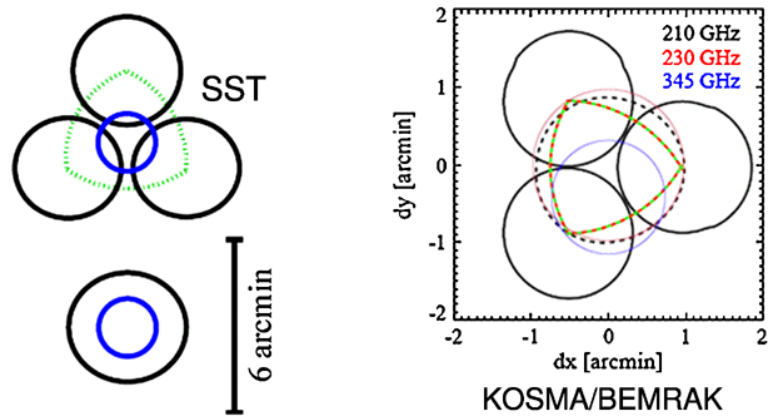

Fig. 1 Beam patterns for SST (left) and KOSMA/BEMRAK (right). SST has six separate beams on the sky: the four larger beams (black) shown are at $212 \mathrm{GHz}$, with three beams three forming a triangle around the target region and one beam in a reference location $6^{\prime}$ away. The two smaller beams are at $405 \mathrm{GHz}$ (blue), with a primary beam usually centered on the target location and a reference beam $6^{\prime}$ away at the reference location. The dashed green line indicates the region within which source location at $212 \mathrm{GHz}$ can be carried out. At KOSMA, the standard 230 and $345 \mathrm{GHz}$ SIS receivers are centered on the target region ( $345 \mathrm{GHz}$ is offset slightly) while three $210 \mathrm{GHz}$ beams in the BEMRAK receiver form a triangle pattern centered on the target location. The dark dashed circle indicates the fourth BEMRAK beam synthesized coherently from the three $210 \mathrm{GHz}$ receivers

At wavelengths around a millimeter and shorter, interferometer observations are difficult and a single-dish approach is far more economical. However, a single-dish telescope with a single receiver has limited mapping ability and is subject to sky transparency fluctuations and noise. Chopping (e.g., Turon and Léna 1970; Hudson 1975) or multiple feeds (e.g., Herrmann et al. 1992) help with these problems and also permit rudimentary imaging or source centroiding. The multi-feed technique was adopted by both the 1.5 meter Solar Submillimeter-wave Telescope (SST) at El Leoncito, Argentina (2550 m altitude), and the 3-meter Kölner Observatorium für Submillimeter und Millimeter Astronomie (KOSMA) telescope at Gornergrat, Switzerland (3135 $\mathrm{m}$ altitude), so far the only telescopes used to detect flares in the 200-400 GHz submillimeter range. In the following sections we discuss the observing techniques utilized by these telescopes and the issue of flux calibration that is critical for understanding the spectra of solar flares at millimeter-submillimeter wavelengths.

\subsection{The solar submillimeter-wave telescope}

SST is a joint Brazil-Argentina project located at high altitude in El Leoncito National Park in Argentina, $100 \mathrm{~km}$ north of the city of Mendoza and in a mountain range to the east of, and in the rain shadow of, the high Andes. The 1.5-meter dish is a Cassegrain design inside a GoreTex radome, with the receiver package containing feeds for 212 and $405 \mathrm{GHz}$ ( $8.5 \mathrm{GHz}$ bandwidth) located at the Cassegrain focus.

The full-width-half-maximum (FWHM) beam size of the $1.5 \mathrm{~m}$ dish is about $240^{\prime \prime}$ at $212 \mathrm{GHz}$ and $120^{\prime \prime}$ at $405 \mathrm{GHz}$. In order to locate the flare source within the fieldof-view and thus to correct measured fluxes for the primary beam response of the telescope, SST has six separate feed horns: four operating at $212 \mathrm{GHz}$, and two at $405 \mathrm{GHz}$. The arrangement of the beams on the sky is shown in Fig. 1. Three of the 
$212 \mathrm{GHz}$ beams are arranged around the observing target in a triangular pattern with spacing equal to the beam FWHM. The fourth $212 \mathrm{GHz}$ beam is used as a reference source and is directed some $6^{\prime}$ away from the target location. A single $405 \mathrm{GHz}$ beam is usually centered on the target location, while a $405 \mathrm{GHz}$ reference beam is coincident with the $212 \mathrm{GHz}$ reference beam. Under the assumption that the flare source is compact on the scale of the $212 \mathrm{GHz}$ beam size, the antenna temperatures of the three $212 \mathrm{GHz}$ beams, together with knowledge of the individual beam shapes, can be used to determine the location and flux of the flare source at $212 \mathrm{GHz}$ as a function of time. The correction factor to be applied to the $405 \mathrm{GHz}$ measurements to compensate for its offset from the center of the power pattern of the telescope beams is determined by assuming that the $405 \mathrm{GHz}$ location is the same as the $212 \mathrm{GHz}$ location.

\subsection{KOSMA/BEMRAK}

Unlike SST, the KOSMA telescope was not originally designed for solar work, but rather for submillimeter observations of galactic star-forming regions. Initial observations with KOSMA used the standard dual-channel single-beam cooled SIS receivers operating at 230 and $345 \mathrm{GHz}$ simultaneously (Lüthi et al. 2004b). The $3 \mathrm{~m}$ dish has beam sizes of $120^{\prime \prime}$ and $90^{\prime \prime}$ at 230 and $345 \mathrm{GHz}$, respectively. However, it was realized that with a single beam it was difficult to identify the true source location, and thus correction for the distance of the source from the pointing center of the dish, necessary to take out the effect of the primary beam response on the apparent source flux, could not reliably be applied.

In order to carry out observations without this limitation, a multi-beam receiver package, the Bernese Multibeam Radiometer for KOSMA (BEMRAK), was constructed (Lüthi et al. 2004a). BEMRAK uses three uncooled $210 \mathrm{GHz}$ Schottky mixers (1 GHz bandwidth), and it and the 230/345 GHz SIS receivers are used simultaneously by placing both at the same Nasmyth port and feeding both using a beam splitter. The arrangement of the beams on the sky is shown in Fig. 1. In addition, BEMRAK "synthesizes" a fourth beam from the other three, centered on the target region. This is achieved by using a single local oscillator to drive the down-conversion mixers in each of the three receivers, ensuring coherency between the signals. The three separate signals are then summed coherently to form the synthesized beam (with the system set up such that the amplitude gains and phase path lengths of the three signals are identical to within suitable limits). The half-power beam width (equivalent to FWHM) of KOSMA at $210 \mathrm{GHz}$ is $100^{\prime \prime}$, while the synthesized fourth beam has an effective half-power beam width of $125^{\prime \prime}$ (the broadening of the synthesized beam is due to small phase and amplitude differences between the signals in the three individual beams that affect their coherent sum). With four beams, the size of the source can be determined in addition to the corrected flux and location of the source.

\subsection{Calibration}

There are two main paths to calibration of the single-dish data, and these will be discussed briefly here. The final step is the correction for the offset of the flare source from the center of the power pattern of the primary beam of the antenna, discussed further in Sect. 2.4.3. This requires 
- detection of the event in at least three beams;

- knowledge of the relative calibration of each of the receivers used to detect the source (at $212 \mathrm{GHz}$ for SST and $210 \mathrm{GHz}$ for BEMRAK); and

- knowledge of the shape of the primary beam response of the telescope-feed combination for each receiver.

The multi-beam method must determine four unknowns, namely position (latitude and longitude in heliographic coordinates), flux and equivalent source size of the emitting source. Thus, at least four independent observations are needed; these are provided by the temperatures observed by four different beams. This is discussed in detail by Giménez de Castro et al. (1999), who present an analytical method to solve the system assuming identical Gaussian beams. They also show that even in the case of only three independent observations (three beams), an upper bound limit for the source size may be provided. Later, Lüthi et al. (2004a) presented a computational method which takes into account actual beam shapes. If the source is known to be much smaller than the beam size, it can be considered point-like, and then only three unknowns are to be determined (Herrmann et al. 1992; Costa et al. 1995).

Very accurate measurement of the beam shapes for each beam is necessary for good determination of locations. Due to the fact that a source could be in any of the sidelobes of the single-dish primary beam pattern (i.e., the peaks in the response beyond the primary beam of the dish), unambiguous results are limited to source locations lying inside the triangle formed by the centers of the three beams. We stress that this method does not provide images: rather, since it has to rely on just three measured data, it is a forward-fitting procedure in which we assume a Gaussianprofile source and the fitted positions are the centroids of flux-averaged sources. The measurements do not contain enough information to determine whether more than one source is emitting simultaneously.

To calibrate the signals in individual beams, one can either proceed from engineering first principles (used for most SST results), or from assumptions about the quiet-Sun signals detected by the beams (used by Lüthi et al. 2004b; Silva et al. 2007).

\subsubsection{Engineering calibration}

The engineering approach is as follows:

- The temperature response of the system, i.e. the conversion between total system temperature and power levels from the receiver measured by square-law detectors, is determined using ambient-temperature and "hot" loads (in the case of SST, at $\sim 300 \mathrm{~K}$ and $430 \mathrm{~K}$, respectively) that fully illuminate the feeds. These two measurements yield both the gain of the system and, in principle, the intrinsic receiver temperature contribution, $T_{\text {rec }}$. The linearity of the response of the receiver is confirmed using occasional measurements of a third load ("cold" liquid nitrogen) and can also be checked using stepped attenuators.

- The total system temperature measured by SST at any time contains contributions from the receiver, the sky, the quiet Sun and any burst in progress (both absorbed by their path through the atmosphere). For each beam $i$ the total system temperature can be represented as 


$$
\begin{aligned}
T_{i}= & T_{\text {rec }, i}+T_{\text {sky }}\left(1-\mathrm{e}^{-\tau / \sin E l}\right) \\
& +\eta_{i} T_{Q S} \mathrm{e}^{-\tau / \sin E l}+\epsilon_{i} T_{\text {burst }, i} \mathrm{e}^{-\tau / \sin E l},
\end{aligned}
$$

where $T_{\mathrm{rec}, i}$ is the effective temperature of the receiver (typically $2000 \mathrm{~K}$ for the $212 \mathrm{GHz}$ receivers at SST), $T_{\text {sky }}$ is the temperature of the atmosphere in the layer where absorption at the observing frequency is strongest (typically $280 \mathrm{~K}$ ), $\tau$ measures the zenith extinction (the opacity of the sky in the zenith direction for the given observing frequency), $E l$ is the elevation of the Sun, $T_{Q S}$ is the quiet Sun temperature (of order $6000 \mathrm{~K}$; measurements of 5900 and $5100 \mathrm{~K}$ at 212 and $405 \mathrm{GHz}$, respectively, were made by Silva et al. 2005), $T_{\text {burst, } i}$ is the temperature contribution of any burst (assumed to be sufficiently compact that most of the primary beam is still filled with the quiet Sun contribution) averaged over the beam area, and $\epsilon_{i}$ is the aperture efficiency for a compact source that fits into the primary beam. This equation assumes that the aperture efficiency for the sky, which fills all the sidelobes of the antenna pattern, is effectively unity. If the beam points at the center of the solar disk then the quiet Sun fills most of the sidelobes of the beam and its contribution has an effective efficiency of order unity, whereas it will have a smaller effective efficiency if the beam targets the limb. To handle this, we include the factor $\eta_{i}$ modifying the quiet Sun contribution for each beam: Melo et al. (2005) find that for a target near disk center $\eta \approx 0.5$ at $212 \mathrm{GHz}$ and 0.6 at $405 \mathrm{GHz}$, while for a target near the limb $\eta$ could be $50 \%$ smaller.

- The opacity of the sky is measured by doing a "sky dip": receiver power is measured as the telescope is moved in elevation from pointing vertically up to almost pointing at the horizon. Elevation and its effect on apparent atmospheric opacity is the main cause of variation in the measured power, and the dependence on elevation can be fitted using a plane-parallel atmospheric model, in order to determine the zenith opacity. At SST, sky dips are done several times per day: it is found that the opacity generally varies slowly unless thunderstorms are present. Typical values for $\tau$ are 0.3 at $212 \mathrm{GHz}$ and 1.5 at $405 \mathrm{GHz}$ (median values: Melo et al. 2005).

- Unfortunately, the aperture efficiencies of the telescope at the observing frequencies need to be known for this method of calibration because the burst contribution comes in through the telescope aperture and is thus modulated by the aperture efficiency, while the receiver temperature, the quiet-Sun contribution (per the assumption above) and the ambient and hot loads do not "see" the aperture. During the initial phase of operation the aperture efficiency of SST was low (less than 0.1) due to imperfections in the dish shape. The dish form was corrected over a number of years, starting in 2001 and continuing through 2006. Aperture efficiencies have small variations from beam to beam at the different frequencies, but typical values at present are 0.35 at $212 \mathrm{GHz}$ and 0.20 at $405 \mathrm{GHz}$.

In effect, the quantities $T_{i}, T_{\mathrm{rec}, i}, T_{\mathrm{sky}}, \tau / \sin E l$ and $\epsilon$ are all measured, so that the temperature contribution of a flare can be determined from

$$
T_{\text {burst }, i}=\frac{T_{i}-T_{\text {rec }, i}-T_{\text {sky }}\left(1-\mathrm{e}^{-\tau / \sin E l}\right)-\eta_{i} T_{Q S} \mathrm{e}^{-\tau / \sin E l}}{\epsilon \mathrm{e}^{-\tau / \sin E l}} .
$$




\subsubsection{Quiet-sun calibration}

Note from (1) that if the telescope has a cooled receiver, such as the KOSMA $230 \mathrm{GHz}$ and $345 \mathrm{GHz}$ receivers, then $T_{\text {rec }} \ll T_{Q S}$ and the total system temperature in the absence of a flare is dominated by the quiet Sun contribution, which we know to be of order $6000 \mathrm{~K}$ to within $\pm 10 \%$, i.e., to much better precision than the absolute calibration obtained with the method described above. Even when $T_{\text {rec }} \approx T_{Q S}$, a simple observation of the sky at the same elevation as the Sun allows one to subtract the receiver and sky contributions: from (1),

$$
\begin{array}{ll}
\text { Sky: } & T_{0, i}=T_{\mathrm{rec}, i}+T_{\text {sky }}\left(1-\mathrm{e}^{-\tau / \sin E l}\right) \\
\text { Quiet sun: } & T_{1, i}=T_{0, i}+\eta_{i} T_{Q S} \mathrm{e}^{-\tau / \sin E l} \\
\text { Burst: } & T_{2, i}=T_{1, i}+\epsilon_{i} T_{\text {burst }, i} \mathrm{e}^{-\tau / \sin E l}
\end{array}
$$

From these three measurements we can derive the burst contribution to the total system temperature as follows:

$$
T_{\text {burst }, i}=\frac{T_{2, i}-T_{1, i}}{T_{1, i}-T_{0, i}} \frac{\eta_{i}}{\epsilon_{i}} T_{Q S} .
$$

SST normally carries out a number of scans across the solar disk each day: the peak response in the scan in each beam can be used as the values $T_{1, i}$ needed above, independent of the power levels when the beams are pointing at the active region target, and as long as conditions do not change greatly between disk scans the calibration parameters should be appropriate for the target region measurements. The advantage of this formulation is that the opacity of the sky no longer appears, and it involves ratios of known $\left(T_{Q S}\right)$ or measured $\left(T_{2, i}, T_{1, i}, T_{0, i}\right)$ quantities. These measurements all see the same atmosphere and other effects, so the ratios remove these and other instrumental effects from the result. The method does, however, still involve the correction $\eta_{i} / \epsilon_{i}$, which has some uncertainty, particularly if $\epsilon_{i}$ is small. A problem with this method, however, occurs when the target region is close to the solar limb and not all beams are uniformly illuminated by the quiet Sun. In effect, $\eta_{i}$ is strongly position-dependent, and therefore poorly determined in such a case.

The approach described here is essentially the idea used in calibration by, e.g., Lüthi et al. (2004a) with KOSMA/BEMRAK and Silva et al. (2007) with SST. Lüthi et al. (2004a) estimate uncertainties of order $20 \%$ in the flux density of the SOL200310-28 flare (which was on the solar disk, not far from disk center) at $210 \mathrm{GHz}$. An example of SST data resulting from application of this calibration technique is shown in Fig. 2. The plot shows light curves of the four $212 \mathrm{GHz}$ beams during an observation of an active region very close to disk center (see Fig. 3) on 2007 November 16. The data have been calibrated exactly as described above, with no additional corrections applied. The calibration data were determined using scans across the solar disk (Fig. 3), of which there were seven, roughly an hour apart and lasting about 20 seconds each. The disk-center temperature was taken to be $6200 \mathrm{~K}$ at $212 \mathrm{GHz}$. The longer data gaps visible in Fig. 2 correspond to times when both sky dip observations and disk scans were taken. The fitted opacities during the period shown were all in the range $0.08-0.09$ at $212 \mathrm{GHz}$ (compared to $0.4-0.5$ at $405 \mathrm{GHz}$ ), while fitted 
Fig. 2 The solar contributions to each beam of SST on 2007 November 16 (during a non-flaring period; beam location see Fig. 3), derived using the "quiet-Sun" calibration technique. The four $212 \mathrm{GHz}$ beams are shown (as labeled) at a sampling rate of order 40 ms. A known small-amplitude instrumental oscillation with a period of order $7 \mathrm{~min}$ has been removed from Beam 1. Note that the temperature increase of beam 1 is due to a change in pointing (see Fig. 3)
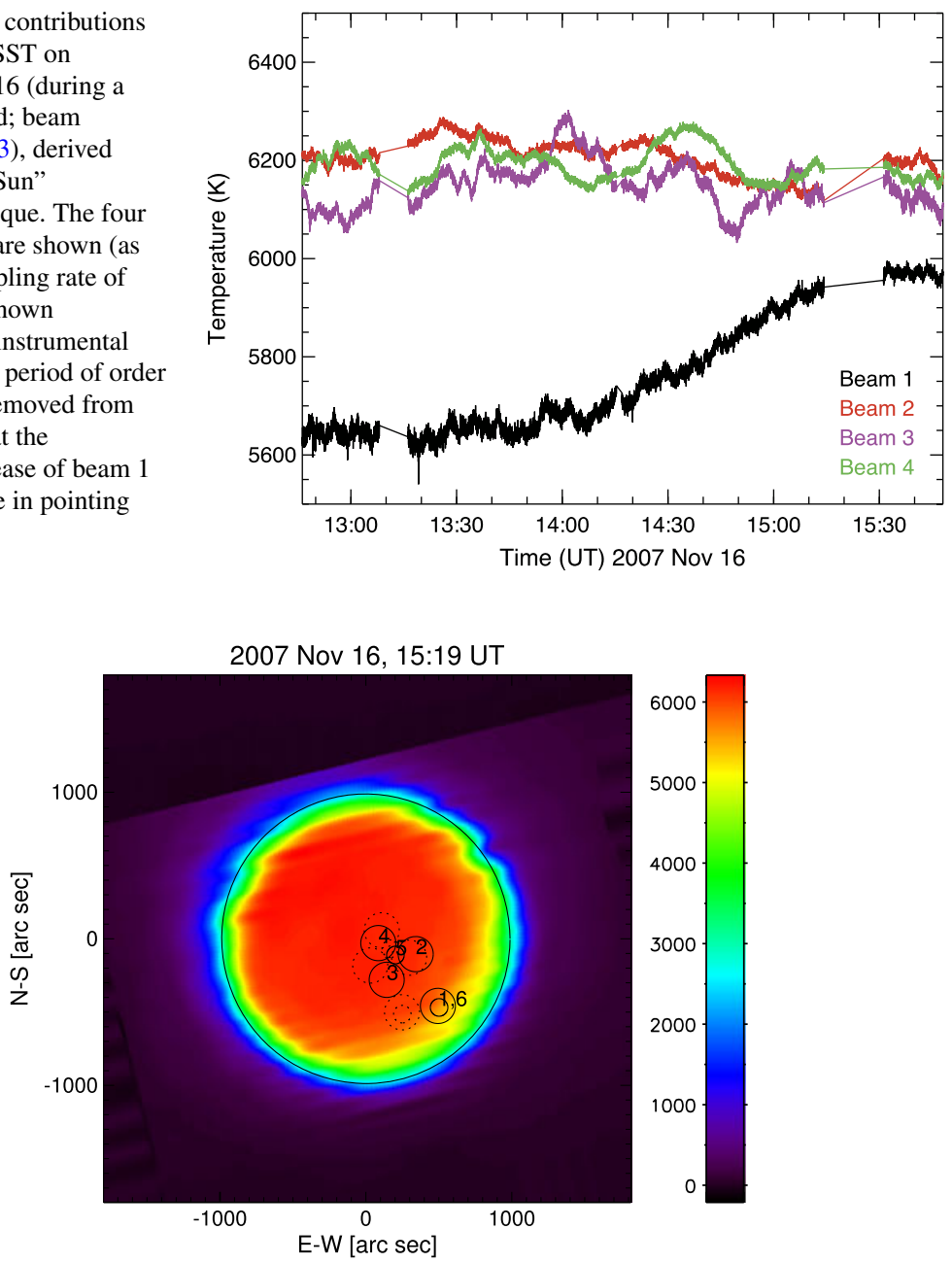

Fig. 3 A solar map obtained from raster scans on 2007 November 16, between 15:15 and 15:23 UT (one of the time gaps in Fig. 2) with Channel $1(212 \mathrm{GHz})$. The map is calibrated as a temperature excess above the sky level (i.e., $T_{\text {sky }}$ is assumed to be zero), on the color scale shown at the right. The circles show the various beams at two different times: 13 UT (solid circles) and 15:40 UT (dotted circles). While the beams rotate relative to the solar disk, the position of the beams may change relative to the limb, varying $\eta_{i}$ and, therefore, changing the observed temperature (cf. Fig. 2)

receiver temperatures varied from $1300 \mathrm{~K}$ on beam 2 to $2900 \mathrm{~K}$ on beam 3 . The calibrated solar temperatures of three of the four beams are consistent with each other, and with the assumed disk-center temperature of $6200 \mathrm{~K}$, to within $2 \%$, while the beam 1 that is pointed closer to the limb (see Fig. 3) gives a lower temperature by up to $10 \%$. No flares occurred in the period shown, so the variation seen in each beam is due to hardware drifts or changes in the atmospheric opacity: a $1 \%$ change in opacity can produce a $60 \mathrm{~K}$ change. Several timescales can be seen in the data. Over short timescales (less than 1 minute) the noise level in the data (at the sampling 
rate of $40 \mathrm{~ms}$ ) is of order $4 \mathrm{~K}$, corresponding to a flux of order $1 \mathrm{SFU}$. But over longer timescales the instrumental variations and atmosphere dominate, producing fluctuations an order of magnitude larger. Further, the levels in the beams need to be balanced before attempting source location fitting for any burst that occurs, and the effective flux limit for this application is somewhat higher than the single-beam noise levels. Later in the day, after the period shown, the atmospheric conditions worsen and fluctuations are more severe. When both calibration methods can be applied, they serve as a check on each other. In the case of Fig. 2, the engineering calibration yields levels very similar to the quiet-Sun calibration results for suitable aperture efficiencies, with essentially identical time variations.

\subsubsection{Determination of the flux, location and source size}

Once $T_{\text {burst }, i}$ is determined for every beam, the multi-beam method must be applied in order to allow for the offset between the actual burst location and the centroids of each of the beams and thus determine the total burst flux and its position. First, position and source size must be determined. Bearing in mind that the observed $T_{\text {burst }, i}$ in detector $i$ is the convolution of the beam response $B_{i}$ and the source flux distribution on the sky, $S$, we have

$$
T_{\text {burst }, i}=S\left(A z_{s}, E l_{s}, \sigma_{s}\right) \otimes B_{i},
$$

and we have to invert Eq. (5) to find $S$. Here the function $S\left(A z_{s}, E l_{s}, \sigma_{s}\right)$ that represents the source is always assumed to be Gaussian, with a center at $\left[A z_{s}, E l_{s}\right]$ and with a Gaussian width $\sigma_{s}$ (i.e., $S \propto \exp \left(-\phi^{2} / 2 \sigma_{s}^{2}\right)$ where $\phi$ is the angular distance from the source center on the sky); and $B_{i}$ are the set of functions that represent the response of each beam. When beams are considered Gaussians and of the same aperture efficiency $\epsilon_{i}$, an analytical and straightforward method can be used (Giménez de Castro et al. 1999). A different approach is the matrix representation of the beams that uses a numerical method to obtain $S$ (Lüthi et al. 2004a). Once positions are determined, they can be used to determine the position-corrected temperature of the burst. For identical Gaussian beams this correction is carried out via

$$
T_{\text {burst }}=T_{\text {burst }, i} \times \exp \left(\frac{\left(A z_{i}-A z_{s}\right)^{2}+\left(E l_{i}-E l_{s}\right)^{2}}{2 \sigma_{e}^{2}}\right),
$$

where $A z_{i}$ and $E l_{i}$ are the azimuth and elevation of the projected positions on the sky of beam $i$; and $\sigma_{e}^{2}=\sigma_{b, i}^{2}+\sigma_{s}^{2}$ is the convolution of the size of the beam and the size of the source, with $\sigma_{b, i}$ the Gaussian width of beam $i$. Four measurements and knowledge of each $\sigma_{b, i}$ are needed to determine $S, A z, E l$ and $\sigma_{s}$; when measurements from only three independent beams are available, we must assume that $\sigma_{s} \ll \sigma_{b, i}$ and neglect the source size.

This is the end of the calibration process. We have now an atmospheric and position-corrected antenna temperature $T_{\text {burst }}$ determined from the independent observations of the different beams, with which we can determine the flux by the standard relation

$$
F=\frac{2 k_{B} T_{\text {burst }} v^{2}}{c^{2}} \Omega_{\text {beam }}
$$


where $\Omega_{\text {beam }}$ is the solid angle occupied by the beam (recall that $T_{\text {burst }}$ is averaged over the beam area), $k_{B}$ is the Boltzmann constant, $v$ is the observing frequency and $c$ is the speed of light. This procedure can give us the flux, location, and in some cases the size of the emitting source at each measured time, i.e., with the time resolution at which the data are acquired, normally better than $100 \mathrm{~ms}$. However, in some events, only one or two beams observe the burst emissions and neither the flux nor position can be determined unambiguously.

\section{Observations}

\subsection{Normal gyrosynchrotron events}

By normal events we refer to flares where the mm-wave emission shows the falling high-frequency extension of the microwave spectrum, interpreted as the gyrosynchrotron emission produced by the relativistic tail of the non-thermal flareaccelerated electrons. Statistical results from fixed-frequency observations between $1 \mathrm{GHz}$ and $35 \mathrm{GHz}$ show peak frequencies during solar flares typically between 3 and 30 GHz (e.g. Guidice and Castelli 1975; Stähli et al. 1989; Correia et al. 1994; Nita et al. 2004). On this basis the gyrosynchrotron spectrum should decrease with frequency throughout the mm-wave range, possibly being overtaken by the flat-spectrum optically thin thermal emission of hot dense soft-X-ray-emitting plasma at some frequency. We return to this topic in detail in Sect. 4, but Fig. 4 (from Stähli et al. 1989) shows how the spectral peak and the shape of the gyrosynchrotron spectrum change with variations of the physical parameters of the source.

About half of the submillimeter events recorded thus far show the expected spectra decreasing with frequency (entry "No" in the "THz" column of Table 1). This suggests that these events simply show the ordinary high-frequency tail of the microwave gyrosynchrotron emission. The event with the best frequency coverage is shown in Fig. 5. For this X-class flare (SOL2002-08-30T13:28) only the $212 \mathrm{GHz}$ observations detect the flare while the $405 \mathrm{GHz}$ observations provide an upper limit. The high-frequency extension of the gyrosynchrotron emission indicates the presence of relativistic electrons. Combined radio-HXR studies (e.g., see White et al. 2011, and references therein) revealed that the electron spectrum inferred from $\mathrm{cm}-\mathrm{mm}$ observations is harder than that inferred from the HXR measurements. This indicates that there is a break in the flare-accelerated electron spectrum around a few hundred $\mathrm{keV}$ with a harder/flatter electron spectrum at higher energies (e.g. Giménez de Castro et al. 2009). Evidence for such a break at photon energies in the 400-1000 keV range has been obtained for a few (different) $\gamma$-ray events (Trottet et al. 1998, 2000; Vilmer et al. 1999; Silva et al. 2007).

\section{2 $\mathrm{THz}$ events}

Besides the events showing a "normal" gyrosynchrotron spectrum during the impulsive phase, there have been six flares that exhibit a second component with a spectrum increasing with frequency from $\mathrm{mm}$ to sub-mm wavelengths. The "normal" events 

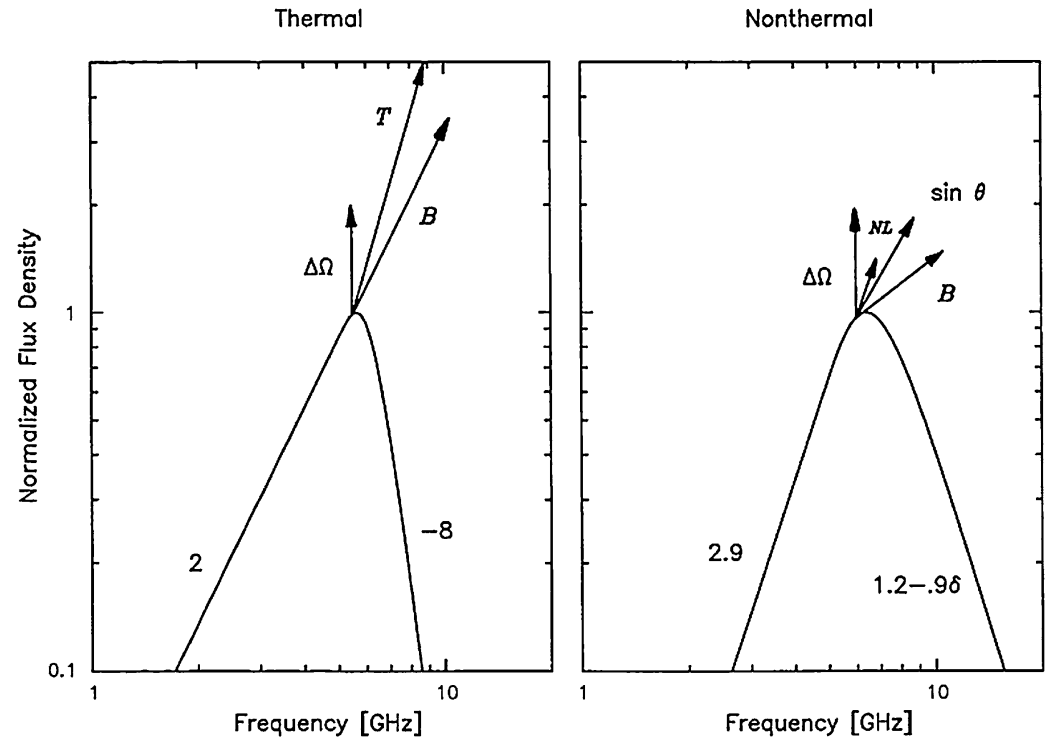

Fig. 4 Parameter dependences of a simple gyrosynchrotron source (from Stähli et al. 1989). The arrows show the effect on peak frequency of factor-of-two changes in the indicated parameters: temperature $T$, magnetic intensity $B$, angle to field $\theta$, solid angle of source $\Delta \Omega$, and length scale $N L$
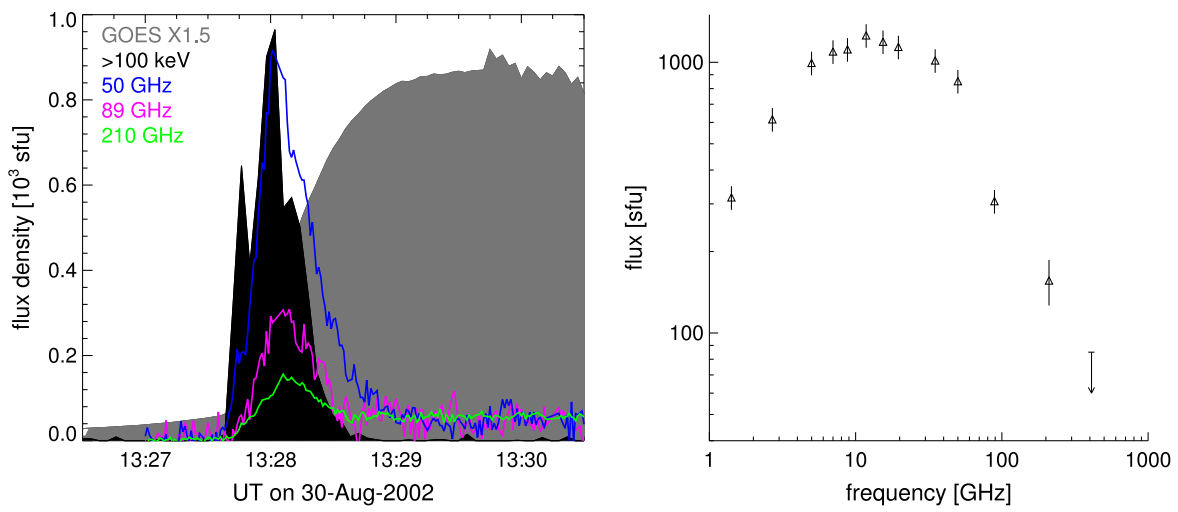

Fig. 5 Left: Radio flux densities at different frequencies (with the GOES SXR and RHESSI HXR flux given in gray and black, respectively); Right: radio spectrum during peak time. The smooth gray curve in the left represents the GOES soft X-ray channel

were associated with SXR flares ranging from M-class to X6, while the four events with the most distinct high-frequency components were large (X6 and higher), eruptive two-ribbon events (Fig. 6). Their time profiles are compared in Fig. 7 with hard and soft X-rays, and spectra from $\mathrm{cm}$ to sub-mm waves are plotted at selected times. Since the results are affected by the observational technique including atmospheric opacity fluctuations, we re-evaluated the calibration and made a new error analysis for 
Table 1 Summary of SST and KOSMA events giving the IAU event identifier, the Active Region number (AR), flare position, GOES class, presence of a THz component (THz), plus the zenith opacity of the sky at $210 \mathrm{GHz}\left(\tau_{210}\right)$ and $405 \mathrm{GHz}\left(\tau_{405}\right)$ at the time of the observation, and the resulting correction factor $f$. The KOSMA events are given in italic; the only event observed by both instruments is given in bold

\begin{tabular}{|c|c|c|c|c|c|c|}
\hline IAU identifier & $\mathrm{AR}$ & Pos & GOES & $\mathrm{THz}$ & $\tau_{210} \& f_{210}$ & $\tau_{405} \& f_{405}$ \\
\hline SOL2000-03-22T18:44 & 8910 & N14W57 & $\mathrm{X} 1.1$ & No & & \\
\hline SOL2001-04-06T19:19 & 9415 & S21E31 & X5.6 & $?$ & & \\
\hline SOL2001-04-12T10:28c & 9415 & $S 21 E 31$ & $X 2.0$ & Yes & & \\
\hline SOL2001-08-25T16:32d & 9591 & S17E34 & $\mathrm{X} 5.3$ & No & & \\
\hline SOL2001-11-28T16:34 & 9715 & N04E19 & M6.9 & No & & \\
\hline SOL2002-08-30T13:28f & 10095 & N15E74 & $\mathrm{X} 1.5$ & No & & \\
\hline SOL2002-09-10T14:53 & 10105 & S10E43 & M3.2 & No & & \\
\hline SOL2002-12-20T13:18 & 10226 & S28W18 & M6.8 & Yes & & \\
\hline SOL2003-10-27T12:31 ${ }^{\mathrm{i}}$ & 10486 & $S 17 E 25$ & M6.7 & No & & \\
\hline SOL2003-10-28T11:10 j & 10486 & $S 16 E 08$ & $X 17.2$ & Yes & & \\
\hline SOL2003-11-02T17:17 & 10486 & S17W63 & $\mathrm{X} 8.3$ & Yes & $0.72 \& 3.4$ & $2.3 \& 39$ \\
\hline SOL2003-11-04T19:44 ${ }^{1}$ & 10486 & S19W83 & $>\mathrm{X} 28$ & Yes & $0.21 \& 1.4$ & $1.4 \& 4.7$ \\
\hline SOL2004-10-30T11:44 ${ }^{\mathrm{m}}$ & 10691 & N13W18 & $\mathrm{X} 1.2$ & No & & \\
\hline SOL2006-12-06T18:44 ${ }^{\mathrm{n}}$ & 10930 & S06E63 & $\mathrm{X} 6.5$ & Yes & $0.40 \& 1.5$ & $2.6 \& 24$ \\
\hline
\end{tabular}

a Kaufmann et al. (2001), Trottet et al. (2002); ${ }^{\mathrm{b}}$ Kaufmann et al. (2002); ${ }^{\mathrm{c}}$ Lüthi et al. (2004b); ${ }^{\mathrm{d}}$ Raulin et al. (2003, 2004); ${ }^{\mathrm{e}}$ Cristiani et al. (2005, 2007b); ${ }^{\mathrm{f}}$ Giménez de Castro et al. (2009); ${ }^{\mathrm{g}}$ Cristiani et al. (2013); ${ }^{\mathrm{h}}$ Cristiani et al. (2008, 2009); ${ }^{\mathrm{i}}$ Trottet et al. (2011); ${ }^{\mathrm{j}}$ Lüthi et al. (2004a); ${ }^{\mathrm{k}}$ Silva et al. (2007); ${ }^{1}$ Kaufmann et al. (2004, 2012); ${ }^{\mathrm{m}}$ Cristiani et al. (2007a); ${ }^{\mathrm{n}}$ Kaufmann et al. (2009)

Fig. 6 Correlation of $210 \mathrm{GHz}$ flux with GOES 1-8 $\AA$. The uncertainties shown for the former are a uniform $30 \%$; events with $\mathrm{THz}$ components are in red

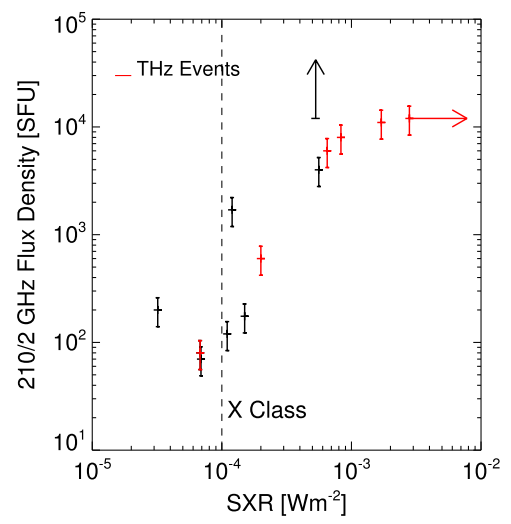

the three SST events discussed below. In the following we cite the 1- $\sigma$ uncertainties derived from our re-analysis.

- SOL2003-10-28T11:10, > X17 (Lüthi et al. 2004a; Trottet et al. 2008): this flare occurred not far from disk center during KOSMA/BEMRAK observations. The HXR emission lasts for more than 20 minutes. The event began with multiple strong peaks over a four-minute interval, followed by a more gradual component 

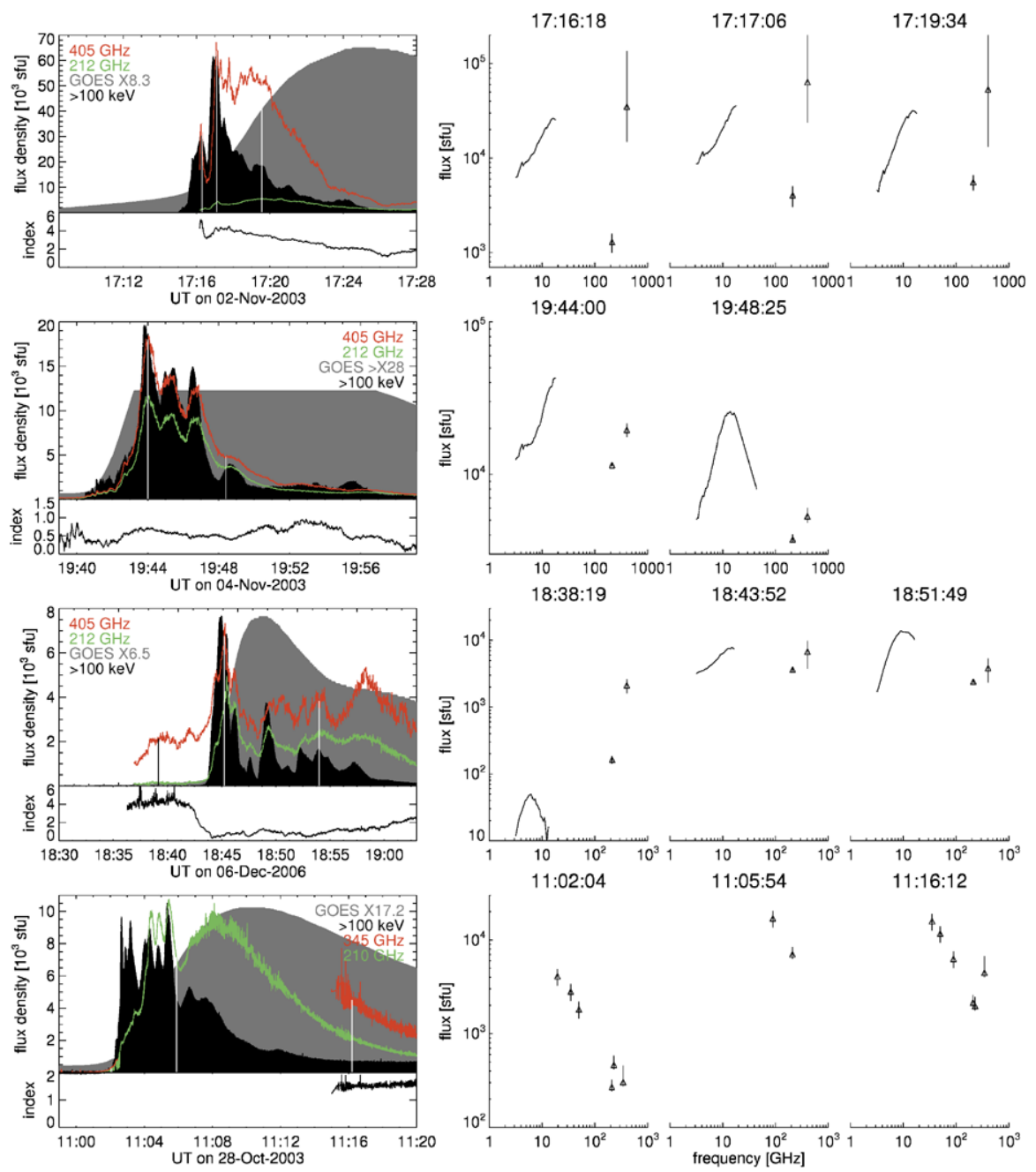

Fig. 7 The four best "THz events" at time of writing: left: normalized linear-scale light curves, and at the bottom the variation of the spectral index inferred from the 405/212 GHz ratio; right: spectra. The smooth gray curves represent the GOES soft X-ray channel; the black curves are the HXR flux from RHESSI, except for the event SOL2003-10-28 where INTEGRAL data is shown. Vertical lines on the light curves designate the times of the individual spectra. For the well-observed event SOL2003-10-28 (bottom panels) the plotted frequencies are 19.6, 35, 50,89.4, and $230 \mathrm{GHz}$, with the $89.4 \mathrm{GHz}$ point appearing only in the second and third frames

and a slow decay. The $210 \mathrm{GHz}$ emission included a slowly varying and a timeextended component $(\sim 11: 00$ UT to $>12: 00$ UT), and three peaks $(\sim 11: 03: 50-$ 11:06 UT) with typical half-power duration of about $20 \mathrm{~s}$ matching those seen in HXR. The HXR peaks at the beginning ( 11:00-11:03:50 UT) of the event have $210 \mathrm{GHz}$ counterparts which can hardly be seen on the figure because they are faint (high frequency part of the decreasing gyrosynchrotron spectrum as shown by the radio spectrum at 11:02:4 UT) and thus show little contrast with the slowly 
varying emission. The $210 \mathrm{GHz}$ gradual component is similar in morphology to, but longer than, that observed in the case of the SOL2003-11-02 flare. Emission peaks at $210 \mathrm{GHz}$ corresponding to HXR sub-peaks seen on top of the smooth HXR emission are also observed. Observations at $345 \mathrm{GHz}$ are not available until later (11:16 UT) in the decay phase. Lüthi et al. (2004a) quote a "worst case" uncertainty of $(+100,-30 \%)$ at $345 \mathrm{GHz}$. Even for this case, the positive slope remains.

- SOL2003-11-02T17:25, X8.3 (Silva et al. 2007): this X8 flare occurred just inside the south-west limb. SST data starting at the first peak in the hard X-rays show initially a very large ratio of $405 \mathrm{GHz}$ flux to $212 \mathrm{GHz}$ flux. The $>100 \mathrm{keV} \mathrm{HXR}$ emission has an overall duration of about $10 \mathrm{~min}$, comprising two main peaks followed by a gradual decay with several superposed minor peaks. The submillimeter emission $(405 \mathrm{GHz})$ matches the HXR emission well until the second main peak. While the HXR count rate drops rapidly afterwards, the $405 \mathrm{GHz}$ emission maintains a high level, with variations that reflect to some extent the minor HXR peaks in the decay phase. The microwave spectra from $\mathrm{cm}$ to sub-mm waves show the two-component behavior throughout the event, with a "normal" gyrosynchrotron spectrum that peaks near or above $20 \mathrm{GHz}$, and a steeply rising $\mathrm{THz}$ component. The observation conditions for this event are not optimal. The effects of atmospheric opacity fluctuations are rather strong. A further problem was related to the beam position: some beams overlapped with the solar limb, which makes the measured signal sensitive to pointing fluctuations. Corrections were derived using a model of the individual beams. The only available calibration coefficients were determined two days later (November 4). As a result, the uncertainties are $7 \%$ $(212 \mathrm{GHz})$ and $35 \%(405 \mathrm{GHz})$ in the flux densities. Nevertheless, these variations do not change the $\mathrm{THz}$ characteristics of the event.

- SOL2003-11-04T19:53, > X17 (Kaufmann et al. 2004): this flare occurred very close to the west limb. The SST and $>100 \mathrm{keV}$ time profiles match each other well: a gradual rise over a few minutes followed by three main peaks within 3 minutes is seen both in the submillimeter data and in the HXR time profile, followed by a decay lasting several minutes. The ratio of HXR to submillimeter emission seems not to vary throughout this event. The spectra show that while the $212 \mathrm{GHz}$ flux density could be part of the decaying "normal" gyrosynchrotron spectrum, the $405 \mathrm{GHz}$ emission is different, despite the similar time evolution. Atmospheric opacity fluctuations contribute to a total uncertainty of $2.5 \%$ and $7 \%$ at 212 and $405 \mathrm{GHz}$, respectively. They were computed using the RMS temperature for the period between 17:50 and 19:00 UT. For fluxes during P1 of 12,000 sfu and 20,000 sfu, these atmospheric fluctuations cannot invert the decreasing microwave spectrum. Even for the weaker P4 pulse, with fluxes of 3,500 and 5,500 sfu at 212 and $405 \mathrm{GHz}$, the atmospheric fluctuations cannot create a positive slope in the spectrum. Hence, this event is one of the clearest examples of an increasing spectrum with frequency.

- SOL2006-12-06T18:47, X6.5 (Kaufmann et al. 2009): this flare occurred just inside the southeast limb, and was interestingly late in the solar cycle. The $>100 \mathrm{keV}$ HXR data show a very strong peak initially, followed by weaker secondary peaks over the next 20 minutes. The SST $212 \mathrm{GHz}$ and $405 \mathrm{GHz}$ time profiles comprise a pre-flare enhancement ( 18:35-18:40 UT), and then successive peaks that are 
overall similar to those at HXR, although differences exist in detail. If the pre-flare emission were also present during the impulsive phase, it should be subtracted from the total spectra to get the spectrum of the impulsive phase source alone. We find that subtracting the pre-flare spectrum does not significantly change the spectral slope during the main peak of the event (18:43:52 UT), but the spectrum shown in Fig. 7 at 18:51:49 UT would change to have a decreasing slope. Even with the (arbitrary) selection of the background as the pre-flare enhancement, the main peak definitely has an increasing spectrum with frequency.

Although the atmospheric transmission conditions were better than for the 2-Nov-2003 event, strong atmospheric fluctuations occurred during the event. Opacity at $405 \mathrm{GHz}$ was 7 times bigger than at $212 \mathrm{GHz}$, whereas the usual ratio is near 4.5. Opacity fluctuations were of the order of $20 \%$, as compared to a few percent in the other cases. This results in variations up to $8 \%$ and $70 \%$ in flux densities at 212 and $405 \mathrm{GHz}$. These fluctuations were not included in the original publication, from which the spectra in Fig. 7 are drawn. In the most extreme case the uncertainties of the opacities (see Eq. (2)) could introduce the THz upturn in the impulsive (18:43:52 UT) and post-impulsive (18:51:49 UT) phases of the event, but not in the pre-impulsive phase (18:38:19 UT).

The uncertainties given in the SST papers refer to a $30 \%$ uncertainty in flux density which was determined in the early days of the instrument. The actually values may be sometimes larger, but can also be smaller. We have re-analyzed the uncertainties for the major SST THz events and obtained the following overall (including opacity and beam imperfection effects) uncertainties: SOL2003-11-02 has $18 \%$ and $51 \%$; SOL2003-11-04, $4 \%$ and $21 \%$, and SOL2006-12-06 $12 \%$ and $100 \%$ at 212 and $405 \mathrm{GHz}$, respectively.

In summary, the results of our re-analysis were found consistent with those of the individual publications. To emphasize the increasing spectra and the possibility that these events could be even more prominent in the $\mathrm{THz}$ range, we call these flares $\mathrm{THz}$ events. The frequency coverage for these events is unfortunately rather poor, except for the decay phase of SOL2003-10-28, and for the last peak of SOL2003-11-04. The incomplete frequency sampling does not allow discrimination as to whether the $212 \mathrm{GHz}$ contains a contribution from the tail of the microwave spectrum. From the ratio of the time profiles at the two frequencies available, power-law slopes between $\sim 0.3$ and $\sim 5$ are derived (without any attempt to determine and subtract the normal gyrosynchrotron component; Fig. 7). Since the "normal" gyrosynchrotron component is expected to show a monotonically decreasing spectrum with frequency in the $\mathrm{mm}$ wave range, the derived power law indices are lower limits for the spectral indices of the new THz component. This suggests that the actual spectrum of the THz component may be rather steep, in particular for the event SOL2003-11-02 where the power law index from 212 to $405 \mathrm{GHz}$ is $>3$.

\subsubsection{Correlations with other wavelengths}

The THz-events generally reach their peak flux during the rise phase of the flare soft X-ray emission (i.e. during the impulsive phase of the flare), and a general 
Fig. 8 Multi-wavelength light curves for the event SOL2003-10-28T11:10. From top to bottom, the $210 \mathrm{GHz}$ signal, total solar irradiance (TSI; Woods et al. 2006), He II 304 AA time profile (from SOHO/CELIAS), four light curves in the hard $\mathrm{X}$-ray and $\gamma$-ray range between 0.1 and $15 \mathrm{MeV}$ from INTEGRAL

(Kiener et al. 2006), and 11-23 MeV and 60-150 MeV light curves (from SONG)

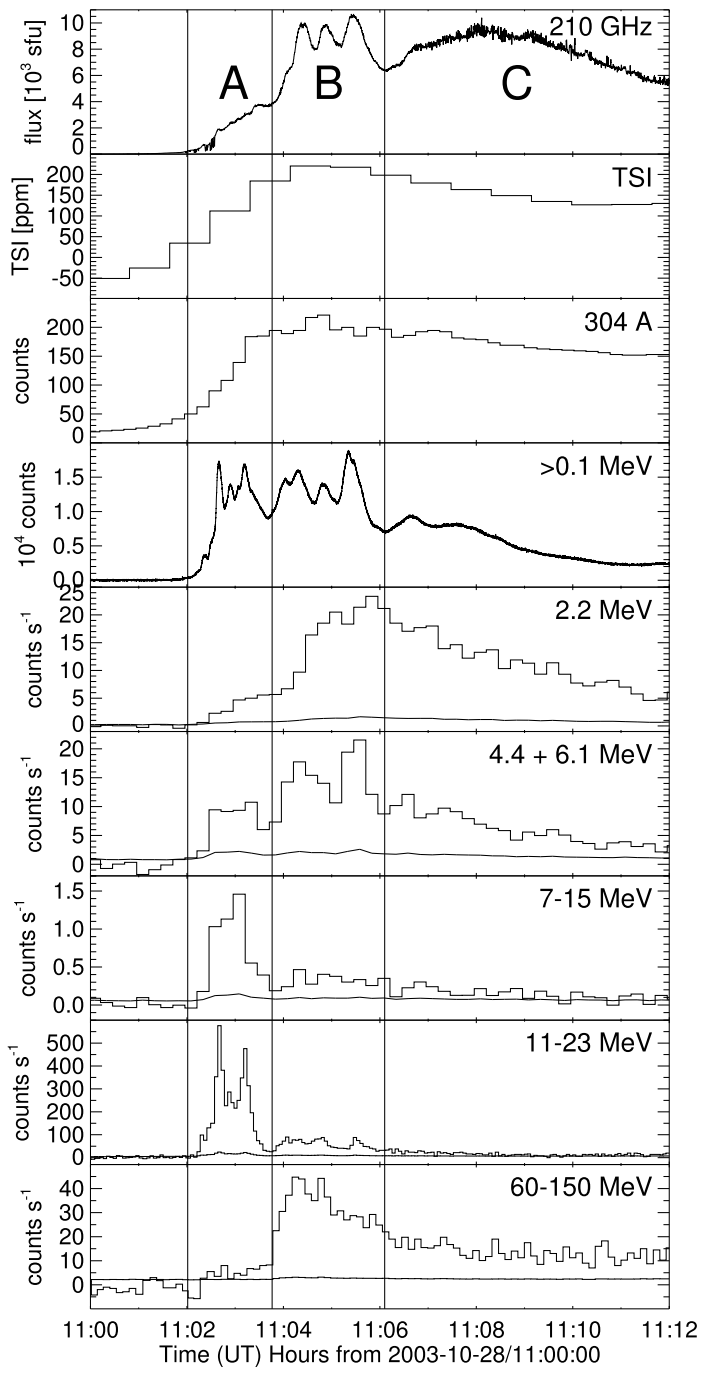

correlation with GOES class exists (Fig. 6). Intense submillimeter emission invariably accompanies hard X-ray emission. Individual peaks are mostly common to the two spectral ranges, and frequently show similar durations (e.g., the flare SOL200311-04 in Fig. 7). Minor peaks can also occur after the soft X-ray peak time (e.g., the flare SOL2006-12-06). The event SOL2003-10-28 additionally shows a longerlasting smooth emission at the submillimeter wavelengths that decays rather slowly, continuing well after the soft X-ray peak, with an e-folding time of order 4 minutes (Trottet et al. 2008, and Fig. 8).

The event SOL2003-10-28 is one of the best-observed major flare events in history. Figure 8 shows its light curves at multiple wavelengths. These include the first-ever detection of a solar flare in the total solar irradiance (Woods et al. 2006). RHESSI obtained hard X-ray and $\gamma$-ray images, and the SONG spectrometer on CORONAS-F 
obtained excellent $\gamma$-ray data (Kuznetsov et al. 2006; Trottet et al. 2008). INTEGRAL obtained observations with unprecedented definition of the 4.4 and $6.1 \mathrm{MeV}$ lines (Kiener et al. 2006). The figure displays clear differences among the various signatures, and there is a remarkable relation of characteristic features at $210 \mathrm{GHz}$ with different hard $\mathrm{X}$-ray and gamma-ray features:

- The hard X-ray emission ( $h v>0.1 \mathrm{MeV}$ ) has two phases (A, B) with strong modulation, followed by a smoothly evolving third phase (C).

- At higher energies phase $A$ is much brighter than phase $B$ in the $11-23 \mathrm{MeV}$ range but phase $\mathrm{A}$ is not detected above $60 \mathrm{MeV}$ whereas phase $\mathrm{B}$ is strong at the highest energies. This corresponds to a steepening of the continuum spectrum above $\sim 20 \mathrm{MeV}$ in phase $\mathrm{A}$.

- In phase B the line emission (e.g., the 4.4 and 6.1 MeV lines) and the line to bremsstrahlung ratio strongly increase compared to phase A. The sharp enhancement of the continuum emission above $60 \mathrm{MeV}$ indicates that the high-energy gamma-ray emission in phase $\mathrm{B}$ is dominated by pion decay photons, i.e. that the primary accelerated particles are protons and alpha particles with energies above 200-300 MeV/nucleon (see also Sect. 4.3.3 below). It should be noted that pion production is still present in phase $\mathrm{C}$ and may continue after 11:12 UT, after which time the gamma-ray measurements were disturbed by the arrival of solar energetic particles.

- The $210 \mathrm{GHz}$ emission evolves smoothly during intervals A and C, but its time evolution is similar to hard X-rays and gamma-rays in phase $\mathrm{B}$. This is the time interval where most of the gamma-ray emission above $\sim 1 \mathrm{MeV}$ is attributable to nuclear interactions of ions.

\subsubsection{Size, positions, and motion of $\mathrm{THz}$ sources}

The small dishes of the multi-beam detection systems provide poor diffractionlimited information on the spatial distribution of the emission (see Sect. 2.4.3). A large source size can result from a single large source, but two well-separated small sources can also mimic a large source. The unique case is when a compact source size is observed. This was the case during the three main peaks of the SOL2003-10-28 event (phase B in Fig. 8). The sources identified in the KOSMA/BEMRAK observations are predominantly compact $\left(<10^{\prime \prime}\right)$ in this case. They are indicated by the red plus signs in the central panel of Fig. 9. Despite the large uncertainty in absolute position (in Lüthi et al. 2004a the uncertainties were conservatively estimated to $<60^{\prime \prime}$ ), the measured location suggests that the $210 \mathrm{GHz}$ emission originates from the southern chromospheric flare ribbon seen in the TRACE UV image. The individual antenna beams did not cover the northern flare ribbon so that one cannot decide if another $210 \mathrm{GHz}$ source existed there. We do not know for sure, however, that the $210 \mathrm{GHz}$ source reveals the $\mathrm{THz}$ component, since the spectral shape at the time of peak emission is not known (Lüthi et al. 2004a). It is possible that the $210 \mathrm{GHz}$ emission is the high-frequency tail of the normal gyrosynchrotron emission. Before and after the compact source is observed, an extended source $\left(\sim 60^{\prime \prime}\right)$ also appears (outlined by the large cross in the top and bottom panels of Fig. 9). For the analysis to generate a compact source during the peak time requires that the flux of the compact 
Fig. 9 Imaging in radio, UV, HXR, and $\gamma$-rays during the three time intervals defined in Fig. 8 for SOL2003-10-28. For each interval, a TRACE image is shown (1600 A for interval A and B; $195 \AA$ for interval C; times are given in the top right corner of each image) with $210 \mathrm{GHz}$ centroid positions overplotted as red crosses (cadence is 10 seconds, increasing symbol size represents time). The average position and source size of the radio emission during each time interval is represented by a thick red cross. The dashed circle gives a rough size of the field-of-view of the radio imaging; radio sources outside this circle are not represented reliably by the derived source position and size. The purple circles in the bottom two images give the flare-averaged $2.2 \mathrm{MeV}$ footpoint point location (from Hurford et al. 2006). The circle size represent 1-sigma uncertainty in the source location. The bottom image additionally shows RHESSI 250-450 keV imaging with 23 arcsec FWHM resolution integrated over the decay phase

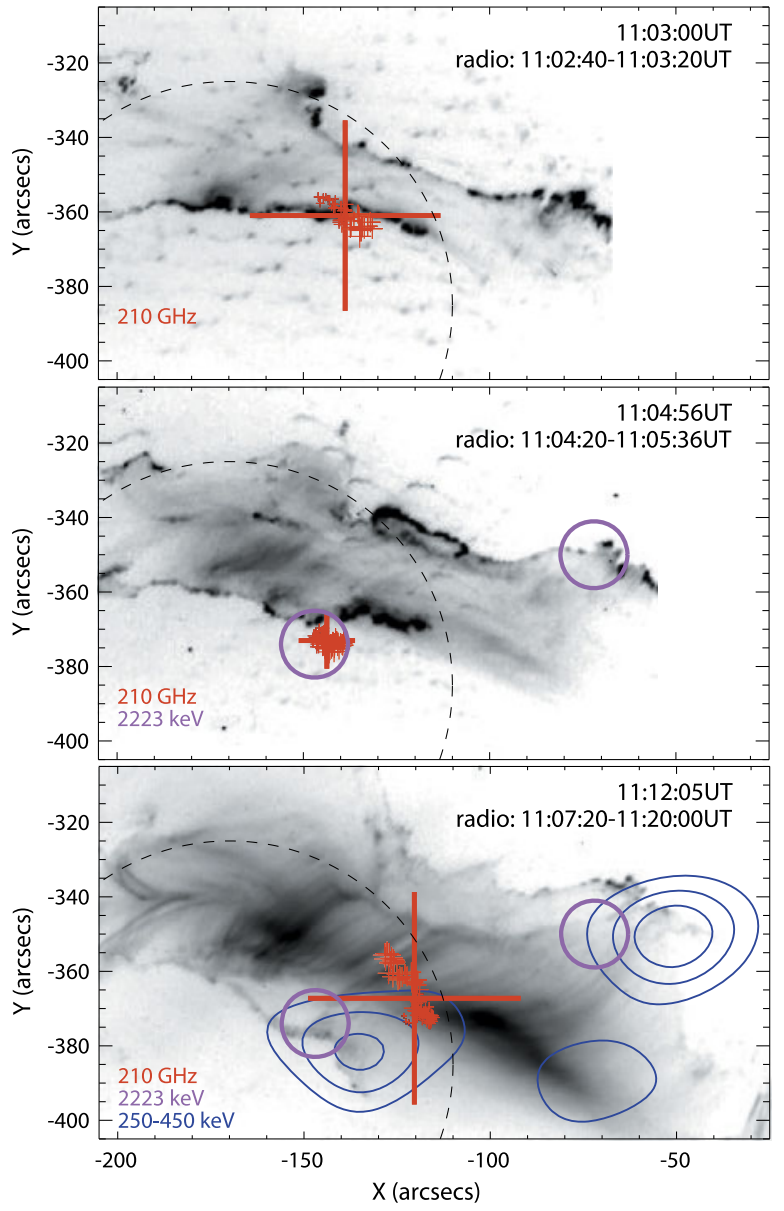

source must dominate that of the extended source during that time interval. This is rather puzzling, as the time profile suggests that the extended source is present at all times.

It is noteworthy that the $210 \mathrm{GHz}$ source is co-spatial with $2.2 \mathrm{MeV}$ emission produced by interacting ions (the sources are indicated by the two circles in the middle and bottom panels of Fig. 9). This finding suggests again, as with the comparison of the timing with the nuclear gamma-ray features, a close connection between the highfrequency emission and nuclear processes during the flare. It is tempting, though far from being demonstrated by the observations, to consider nuclear interactions as a possible origin of the $\mathrm{THz}$ component.

The only direct measurement of a flare source size available to date was produced by scanning a single beam antenna over the flare area (Lüthi et al. 2004b). This observation was during the decay phase of SOL2001-04-12T10:28 (1 hour after the impulsive phase) when the emission mechanism is thermal $\mathrm{f}-\mathrm{f}$ emission. The size at $345 \mathrm{GHz}$ was larger $\left(70 \pm 6^{\prime \prime}\right)$ than that at $210 \mathrm{GHz}\left(42 \pm 20^{\prime \prime}\right)$ possibly indicating two different emission regions at different temperatures (see also Trottet et al. 2011). 

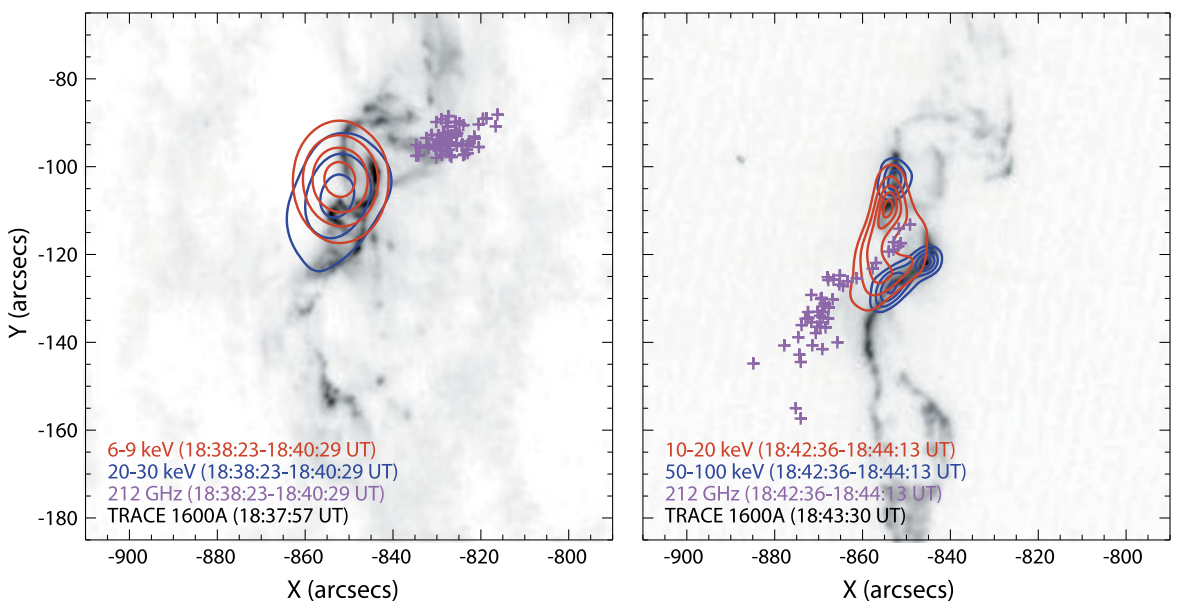

Fig. 10 Time-resolved imaging of SOL2006-12-06T18:47 during (left) the rise phase of the event, and (right) the main impulsive peak. The underlying gray-scale image is a $1600 \AA ̊$ UV continuum image from TRACE, red contours show the location of predominantly thermal soft X-rays, blue contours show higher-energy non-thermal hard X-rays, and the purple crosses show sequential measurements of the location of the $212 \mathrm{GHz}$ source from SST data, as described in Sect. 2.4.3

Fig. 11 Time profiles at $405 \mathrm{GHz}$ (black) and $212 \mathrm{GHz}$ (gray) of the SOL2003-11-04 event observed by SST. The inset shows a 30 -s zoom, with a 1-s running mean flux (smooth curves) and corresponding $\pm 3 \sigma$ levels (dashed)

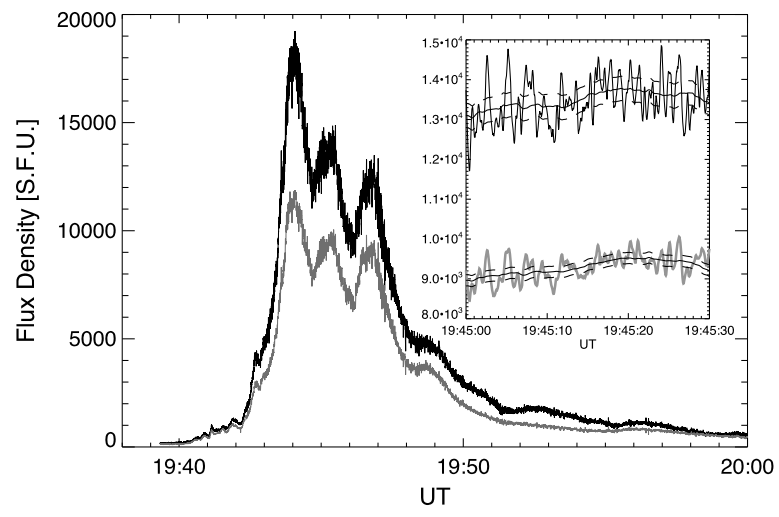

The source locations in all four $\mathrm{THz}$ events show systematic motion, but the motions are not understood and there is no clear correlation with source locations seen at other wavelengths. Kaufmann et al. (2009) report a significant displacement in position of $30^{\prime \prime}$ from the initial phase of the SOL2006-12-06 event (before 18:40 UT) to the main peak (Fig. 10). During the impulsive phase, the THz source moves roughly parallel to the flare ribbons (Fig. 10, right). However, the limited imaging information of a multi-beam system and the relative (5-10") and absolute (up to $30^{\prime \prime}$ ) positional uncertainties could mask possible correlations.

\subsubsection{Fast time variations}

During many mm-sub-mm events, including the THz events, rapid pulsations (at $1 \mathrm{~s}$ period or faster) are observed superimposed on a more gradual flux-density variation 

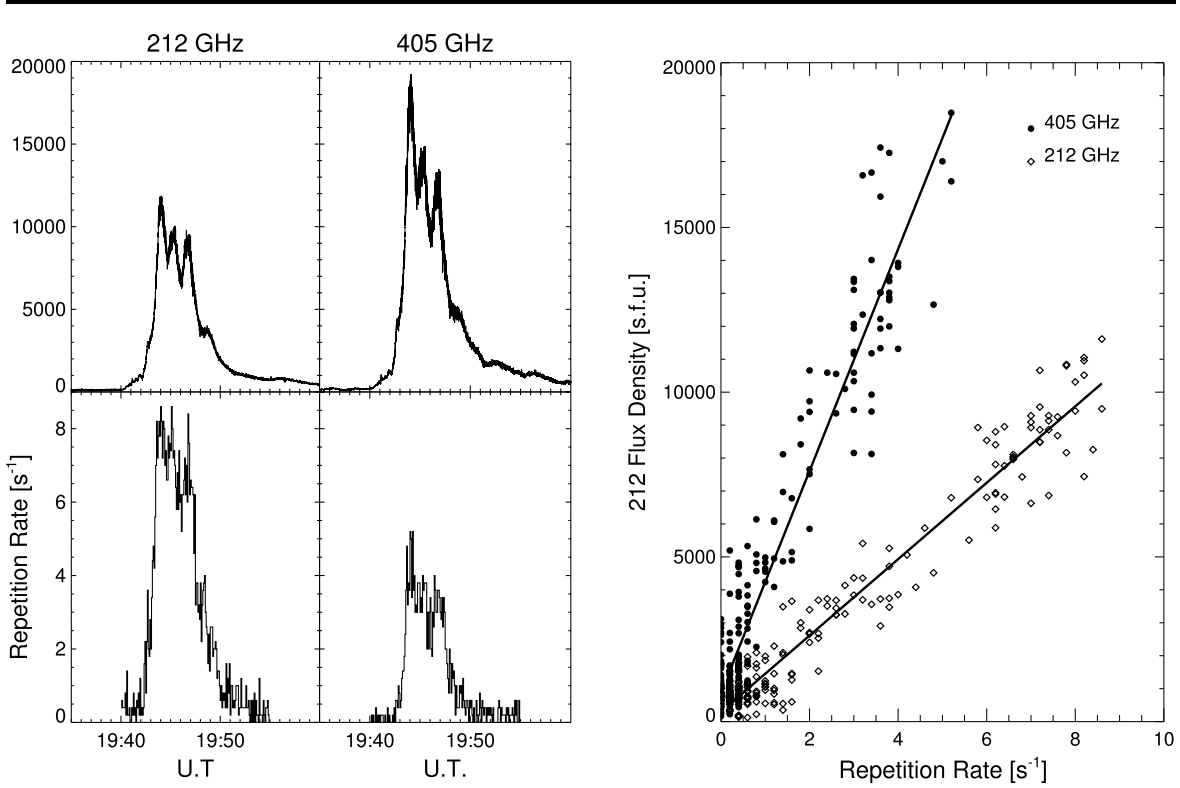

Fig. 12 Left: Top panels show the flux densities at 212 and $405 \mathrm{GHz}$ for SOL2003-11-04, bottom panels show the repetition rate of the fast pulsations observed at these frequencies. Right: the linear relation is obtained when we plot the mean flux density vs. the repetition rate (Kaufmann et al. 2009)

(see the examples in Fig. 11). We term the slow background emission component the "bulk" variation here. These rapid oscillations appear as variations around the mean value of the signal, with temporal scales ranging from a few tens of ms to $1 \mathrm{~s}$. Similar pulsations have sometimes been detected at $\mathrm{cm}$ and $\mathrm{mm}$ wavelengths since the $1980 \mathrm{~s}$ (e.g. Gaizauskas and Tapping 1980; Kaufmann et al. 1980; Raulin et al. 1998). Since the operation of the SST, similar pulses have been observed at sub-mm (Kaufmann et al., 2001, 2002, 2004, 2009; Makhmutov et al. 2003; Raulin et al. 2003; Silva et al. 2007). We note that the direct comparison of $\mathrm{THz}$ and microwave/mm-wave pulse structures has been difficult due to the typical flux saturation at microwaves during powerful events.

Raulin et al. (2003) analyzed the characteristics of these rapid pulsations at 212 and $405 \mathrm{GHz}$ during a normal gyrosynchrotron event observed by SST (SOL2001-08-25). Both the direct-detection method and a wavelet-decomposition analysis showed that the time series contained a variety of scales: several-minute time variations during the whole main event, variations of a few seconds up to $10 \mathrm{~s}$ during the impulsive phase, and superimposed subsecond fast pulses. The positions of the fast pulses, estimated from the temperature excesses recorded with different channels of the SST, were found to be spread within the whole active region, which supports the idea that the primary energy release region is composed of multiple, discrete, and compact sites, highly variable in time (Raulin et al. 2000). Our knowledge of pulse locations will benefit from future spatially resolved observations with new, high-sensitivity, submillimeter instruments (see Sect. 5).

The number of pulses per unit time (the so-called "repetition rate" $r r$ ) varies with time, with a maximum value observed thus far of $8 \mathrm{~s}^{-1}$. The amplitude of the oscil- 
lations $\Delta F$ is also a function of time with values of the order of few hundred SFU. As a flare develops, the system noise $\Delta T_{\text {noise }}$ should increase. However, (Raulin et al. 2003) showed that the pulsation temperature amplitude $\Delta T_{\text {pulse }}>\Delta T_{\text {noise }}$, thus tending to rule out an instrumental explanation for the pulsations. In Fig. 12 we show the main features of the rapid pulsations observed during the SOL2003-11-04 THz event. The left panel shows a comparison between time variations of the repetition rate and the bulk emission, showing an apparent similarity between them. The right panel shows linear relations (for each observing frequency) between the mean flux density of the bulk emission and the repetition rate, with the proportionality coefficients $k_{405}>k_{212}$.

Below we present the main characteristics of the sub-mm pulsations and a comparison with related phenomena at lower frequencies:

- The amplitude the sub-mm pulsations normalized to the bulk emission ("ripple") is observed to be of order 5 to $8 \%$. This is an intriguing result, since extrapolation from previous work suggested a ripple amplitude above $100 \%$. Indeed, during the SOL2003-11-04 THz event, observations at $44 \mathrm{GHz}$ show a ripple of around $80 \%$ (Kaufmann et al. 2009). This suggests that the sub-mm pulsations have a different physical origin.

- The spectral index of the sub-mm pulsations is always positive, with maximum values around 2 (Kaufmann et al. 2001).

- There is a linear relation between $r r$ and the mean bulk emission flux density, i.e., $\left\langle\Delta F_{\text {pulse }}\right\rangle \propto r r$, with a frequency-dependent constant of proportionality. A similar trend is found at low frequencies (Kaufmann et al. 1980; Qin et al. 1996; Raulin et al. 1998).

- Kaufmann et al. (2003) suggest a relationship between sub-mm pulsations and CME launch.

There is no compelling evidence that the observed signatures of the pulsations refer particularly to the sub- $\mathrm{THz}$ or $\mathrm{THz}$ domains. For the purposes of this review, we therefore henceforth restrict our attention to the spectral signatures.

\subsection{Critical assessment of the sub-THz spectra}

We are now in a position to ask the key question: Are the positive slopes in the sub-THz range credible? The assumption that this is the case has motivated this review, and any positive answer will encourage future observations of such phenomena, which we believe to be important for flare physics. The reader should now appreciate the difficulties in obtaining the broad-band photometric precision needed to be sure about the answer, in the absence of true imaging spectroscopy. For SOL200310-28, Lüthi et al. (2004a) quote a "worst case" uncertainty of $(+100,-30 \%)$ at $345 \mathrm{GHz}$. For the SST observations the most critical difficulty is the large correction factors (see Table 1). For both SOL2003-11-02 and SOL2006-12-06 we find that atmospheric opacity fluctuations may have a critical effect on the sub-mm spectrum during part of the event. We must bear in mind that this large uncertainty is only one component of the total uncertainty, although this factor generally is dominant. Nevertheless, the Sun itself provides a well-understood calibration standard, and so 
gross calibration errors are improbable. Indeed the only common observation of a solar flare by SST and KOSMA shows that the flux densities derived using the independent calibration methods of each instrument provide consistent values of the flux densities within about $10 \%$ near $210 \mathrm{GHz}$; these are closer to each other than the uncertainties of the individual measurements (Trottet et al. 2011, their Fig. 1). We note here that this good agreement was achieved despite the fact that the opacities of the SST observations with 0.46 and 1.9 at 212 and $405 \mathrm{GHz}$ were rather high (cf. Table 1).

Generally the available data refer to three flare epochs of non-thermal emission: pre-impulsive, impulsive, and post-impulsive. In each of these epochs we have significant evidence for a THz component; the spectra in Fig. 7 definitely show cases of upturns towards the $\mathrm{THz}$ range, especially by reference to the negative spectral indices present or inferred at lower frequencies. Specifically we note SOL2006-1206 for the pre-impulsive, SOL2003-11-04 for the impulsive, and SOL2003-10-28 for the post-impulsive phases. The existence of these indications in multiple events (though too few!) and from two observatories using different instrumentation, makes a convincing case for the existence of an independent component of emission at the shortest wavelengths.

In summary, the quality of the data at present fully justifies the vigorous theoretical work that we describe in the following sections.

\section{Mechanisms}

Surprisingly many mechanisms have been proposed already for the THz events. One reason for this is the strong connection with high-energy processes that may be fundamental to the physics of energy release and particle acceleration. In the following we touch on the areas of thermal, magnetic, and coherent processes separately. For convenience we summarize the several different emission mechanisms discussed below in Table 2, at the end of this section. At the highest frequencies the solar atmosphere becomes transparent, and the background solar radiation forms in the photosphere. Accordingly we start the discussion of individual mechanisms with thermal processes (see Heinzel and Avrett 2011, for a discussion of these processes in the context of semi-empirical flare model atmospheres).

The theoretical work described below usually starts from the idea that the source is uniform and isotropic, with unique physical parameters. Dropping this assumption in general would make things much more complicated, but there is one basic possibility that needs to be borne in mind. An external absorber could easily interpose itself between the source and the observer, and for the mm-sub-mm continuum this could easily result in a positive slope, depending upon the 3D structure of the absorber. Examples of this are well known in the literature of solar and stellar flares (e.g. Covington 1973; Giampapa et al. 1982).

\subsection{Rayleigh-Jeans tail}

We can first wonder if the THz emission is not simply due to the low-frequency extension of the white-light continuum flare emission. A description of this component 
as a black body should suffice, and predicts a Rayleigh-Jeans tail in the mm-submm range. To quantify this, we consider Hinode SOT (Tsuneta et al. 2008) G-band $(430 \mathrm{~nm})$ observations of the SOL2006-12-06 flare. From the observed increase of G-band emission and the area of the white light flare ribbons (Wang 2009), we estimate the flux density at $405 \mathrm{GHz}$ flux to be only a few SFU, about three orders of magnitude below the observed peak flux. The estimated temperature for this flare was relatively low $(8,000 \mathrm{~K})$ but taking the upper limit of $25,000 \mathrm{~K}$ given by Fletcher et al. (2007) still leads to a discrepancy of about a factor of 100 that could only be resolved if the flaring area is very much larger than is observed in the white-light data.

Silva et al. (2007; see also Trottet et al. 2008; Kaufmann et al. 2009) point out that if the source temperature is of order $20 \mathrm{MK}$, the emitted soft X-ray (SXR) flux would greatly exceed the fluxes observed by the GOES satellites in these events. Similarly, material at $2 \mathrm{MK}$ sufficient to produce the observed submillimeter fluxes would be far brighter than the flare features observed in Fe XII $195 \AA$ A images. These considerations apply to the more general case of a thermal source distributed in height, as discussed in the next section.

\subsection{Thermal free-free emission}

Continuum emission and absorption in the quiet solar atmosphere is dominated by thermal $\mathrm{H}$ II and $\mathrm{H}^{-}$free-free opacity at submillimeter wavelengths (e.g., Vernazza et al. 1981), resulting from collisions by thermal electrons with protons and neutral hydrogen, respectively. During a flare the ionization fraction will increase in some regions, and the thermal $\mathrm{H}$ II free-free emission and absorption then begin to dominate. For a fully ionized plasma with solar abundances for $\mathrm{H}$ and $\mathrm{He}$ the free-free absorption coefficient $\kappa_{f f}$ is given approximately as

$$
\kappa_{f f} \approx \frac{\beta n_{e}^{2}}{T^{3 / 2} \nu^{2}} \mathrm{~cm}^{-1},
$$

where $\beta \approx 0.16$ for plasma temperatures of $0.2-2 \mathrm{MK}$ and $\beta \approx 0.2$ for temperatures of $2-20 \mathrm{MK}$.

\subsubsection{Source size}

As noted previously, the flux density $S_{v}$ can then be written conveniently in terms of the mean brightness temperature $T_{B}$ as in (7),

$$
S_{v}=2 k_{B} \frac{v^{2}}{c^{2}} T_{B} \Omega,
$$

where now $\Omega$ is the solid angle filled by the emitting source(s). For a homogeneous source this can be expressed in SFU as

$$
S_{\mathrm{SFU}}=8.2 \times 10^{-5} T_{6} v_{9}^{2} \theta^{2},
$$

where $T_{6}$ is the mean brightness temperature in $\mathrm{MK}, v_{9}$ is the frequency in $\mathrm{GHz}$ and $\theta$ is the characteristic angular size of an equivalent Gaussian source in arcseconds. Since we can give plausible physical arguments for the likely temperature range of 
the thermal material, we can use this equation to estimate the size of a thermal source of known flux $S$ :

$$
\theta \approx 110 S_{\mathrm{SFU}}^{1 / 2} T_{6}^{-1 / 2} v_{9}^{-1} \operatorname{arcsec} .
$$

For the large flares displaying a $\mathrm{THz}$ component we have $S_{\mathrm{SFU}} \approx 10^{4}$. For a frequency of $300 \mathrm{GHz}$, we then have $\theta \approx 37^{\prime \prime} \times T_{6}^{-1 / 2}$. Thus if such a thermal source is to be optically thick at a transition-region temperature $T_{6} \approx 0.2$, it has to have a large size $\theta \approx 80^{\prime \prime}$; a source with coronal brightness temperature $T_{6} \approx 2$ requires a moderate size $\theta \approx 26^{\prime \prime}$; and a source that is optically thick at a soft-X-ray-emitting temperature $T_{6} \approx 20$ only needs a very small size $\theta \approx 8^{\prime \prime}$.

An inverted spectrum $(\alpha>0)$ between 212 and $405 \mathrm{GHz}$, for example, requires the $405 \mathrm{GHz}$ emission to have an optical depth $\tau_{f f}>0.3$; a spectral index $\alpha=2$ requires $\tau_{f f}>3$. The optical depth is given by $\tau_{f f} \approx \kappa_{f f} L$, where $L$ is the depth of the source. To meet the condition $\tau_{f f}>1$ requires the column emission measure to be such that $n_{e}^{2} L>10^{27} \beta^{-1} v_{9}^{2} T_{6}^{1.5} \mathrm{~cm}^{-5}$. For $405 \mathrm{GHz}$, an optical depth greater than unity requires $n_{e}^{2} L>10^{32}$ for $T_{6}>0.2 ; n_{e}^{2} L>3 \times 10^{33}$ for $T_{6}>2$, and $n_{e}^{2} L>$ $7 \times 10^{34}$ for $T_{6}>20$.

For illustration, we apply the above equations to the SOL2006-12-06 flare to check if the observed $405 \mathrm{GHz}$ emission of $S_{\mathrm{SFU}} \approx 7000$ could be free-free emission from the flare ribbons. For a ribbon area of $2.6 \times 10^{17} \mathrm{~cm}^{2}$ we derive $\theta \approx 7^{\prime \prime}$ and the required ribbon temperature becomes $T_{6} \approx 10.8$. To have an optically thick source further requires $n_{e}^{2} L>3 \times 10^{34}$, giving a lower limit of the emission measure of the flare ribbon of $E M=n_{e}^{2} L A>7 \times 10^{51} \mathrm{~cm}^{-3}$. The required temperature and emission measure correspond to a GOES flare class of X100 from the ribbons alone, well above the observed flux of X3 at the peak time of the $405 \mathrm{GHz}$ emission. Hence, to produce the $\mathrm{THz}$ emission by free-free emission from the flare ribbons, far too strong soft $\mathrm{X}$-ray emission from the ribbons would be produced. A larger source at cooler temperature (e.g. $\theta \approx 80^{\prime \prime}$ and $T_{6} \approx 0.2$ ) remains a possibility, but such an extended source in the chromosphere is not observed at any other wavelengths. While during the decay phase source sizes of the order of $\theta \approx 80^{\prime \prime}$ are observed (e.g. Lüthi et al. 2004b), their temperatures are much hotter than $T_{6} \approx 0.2$. Emissions from flare ribbons that could be at $T_{6} \approx 0.2$ requires a longer ribbon than typically observed (i.e. a ribbon length of $320^{\prime \prime}$ assuming a ribbon thickness of $10^{\prime \prime}$ to account for a source size of $\left.\theta \approx 80^{\prime \prime}\right)$. In summary, during the impulsive phase of flares, thermal free-free emission is unlikely the emission mechanism of the $\mathrm{THz}$ component.

\subsubsection{Source spectrum}

The defining characteristic of the THz events is their inverted spectrum: the flux density increases between 200 and $400 \mathrm{GHz}$ as $S=S_{\circ} \nu^{\alpha}$. The index $\alpha$ is found to range from 0.3 to 5 , though with large uncertainties (see Sect. 3.2) such that the observations may generally be consistent with $\alpha \leq 2$. An isothermal free-free source would have a Rayleigh-Jeans spectrum $(\alpha=2)$, but a more general multithermal one can produce a steeper slope (e.g. Heinzel and Avrett 2011).

The structure of the lower atmosphere during the impulsive phase of a flare remains relatively unknown, but has many important ramifications. One of these is the 
perplexingly broad $511 \mathrm{keV}$ line observed by Share et al. (2004), which, taken at face value, implies a large emission measure at transition-region temperatures. Silva et al. (2007) argue that such a source can be excluded because the implied luminosity is far greater than that inferred by Raymond et al. (2007) for a selection of X-class flares. Raymond et al. point out that the O VI luminosities they measure are consistent with a conventional high-pressure transition region with an electron number density of $\sim 10^{12} \mathrm{~cm}^{-3}$ and a thickness of $100 \mathrm{~km}$. They note, however, that other observations (e.g., Doschek et al. 1977) suggest that number densities of $10^{13}-10^{14} \mathrm{~cm}^{-3}$ from a thinner layer are plausible for large flares.

Returning to our requirements on the column emission measure of material at $400 \mathrm{GHz}$, we find that number densities of a few $\times 10^{12} \mathrm{~cm}^{-3}$ over a thickness of $100 \mathrm{~km}$ are needed to render material at $0.2 \mathrm{MK}$ optically thick at $400 \mathrm{GHz}$, but a density of $10^{13} \mathrm{~cm}^{-3}$ requires a thickness of only $10 \mathrm{~km}$, implying a pressure of order 550 dyne $/ \mathrm{cm}^{2}$. We conclude that if the material at transition-region temperatures has densities in excess of $10^{13} \mathrm{~cm}^{-3}$, then it may be optically thick during large flares and such material could account for the inverted spectrum observed in the $\mathrm{THz}$ component provided $\alpha \leq 2$. We point out, however, that Raymond et al. (2007) consider the power carried by thermal conduction into the transition region and find that it greatly exceeds radiative losses. They conclude that either the conductive power is greatly over-estimated or that the two-dimensional filling factor of the dense UV/EUV-emitting material must be low. If the latter interpretation is correct, it implies that the angular extent that a multi-beam system would measure for a thermal source at $0.2 \mathrm{MK}$ producing a flux as high as $10^{4} \mathrm{SFU}$ at $300 \mathrm{GHz}$ may need to be much larger than the $80^{\prime \prime}$ inferred above. Since the observations do not seem to be consistent with such spatially large sources for the $\mathrm{THz}$ emission, we conclude that this mechanism, though attractive because of its Rayleigh-Jeans spectral distribution, probably does not apply.

The flare SOL2003-11-02 provides the most striking example of a flare with a steep positive slope between 212 and $405 \mathrm{GHz}$ (see Fig. 7). The spectrum is steepest $(\alpha \approx 5)$ at the beginning of the event when a dip occurs in the flux density of both bands, possibly the signature of absorbing material. If the steep positive slope is attributed to cool, absorbing foreground material, the absorber must be large enough to obscure a significant fraction of the $212 \mathrm{GHz}$ source and persist for several minutes.

\subsubsection{Source variability}

The origin of the rapid variations (Sect. 3.2.3) is presently unknown. We have established above that if free-free emission is relevant the bulk emission must originate from a relatively extended source with a transition-region temperature. At these temperatures, the radiative loss function has a value of $\Lambda \approx 4 \times 10^{-22} \mathrm{ergs} \mathrm{cm}^{3} \mathrm{~s}^{-1}$ (e.g. Cox and Tucker 1969). The radiative loss time is $t_{R}=3 n_{e} k_{B} T / \dot{E}_{R} \approx 10^{12} T_{6} / n_{e} \approx$ 25-250 ms for $T_{6}=0.2$, assuming a density in the range of a few times $10^{12}$ $10^{13} \mathrm{~cm}^{-3}$. Thus radiative loss from dense features at transition-region temperatures experiencing quasi-periodic heating episodes would be consistent with the time scales of the observed subsecond variations. Kašparová et al. (2009) have simulated such processes in model atmospheres. However, such rapid heating is unlikely to be spatially extended over scales of many tens of arcseconds, so we would expect it to be a 
highly localized feature. (Raulin et al. 2003) show that the positions of the centroids of discrete subsecond spikes have considerable scatter around the mean position inferred for the bulk of the emission, amounting to 1-2 arcmin. Taking the flux density of a discrete spike at $405 \mathrm{GHz}$ to be $\sim 300 \mathrm{SFU}$, and assuming a temperature $T_{6}=0.2$ or greater implies a source size of $10^{\prime \prime}$ or smaller. We suggest that the fast time variations could be explained by small-scale sources of free-free emission.

\subsubsection{Events with thermal emissions}

SOL2001-04-12T10:28 and SOL2003-10-28T11:10 (see, respectively, Lüthi et al. 2004b and Trottet et al. 2008) provide clear examples of flares with thermal freefree emission detected up to submillimeter wavelengths. More recently, Trottet et al. (2011) extensively discusses free-free emissions seen at 200-400 GHz for the flare of SOL2003-10-27T12:43). For flares SOL2001-04-12 and SOL2003-10-27 the thermal emission last for tens of minutes after the impulsive phase and corresponds to the traditional microwave bursts refer to as "gradual rise and fall" or "post-burst increase" (e.g. Kundu 1965; Hudson and Ohki 1972; Shimabukuro 1972). This thermal emissions consists of two components, an optically thin component from coronal loops at $1-3 \times 10^{6} \mathrm{~K}$ observed in the $10-200 \mathrm{GHz}$ wavelength range, and an optically thick component from chromospheric regions with temperatures in the range $10^{3}-10^{5} \mathrm{~K}$ (Trottet et al. 2011). The size of the emitting regions is rather large, being $\sim 40^{\prime \prime}$ around $200 \mathrm{GHz}$ and $\sim 70^{\prime \prime}$ in the 200 and $400 \mathrm{GHz}$ range, respectively (Lüthi et al. 2004b). The post-impulsive phase of the SOL2003-10-28 flare is somewhat different. It shows simultaneously non-thermal and thermal emission. At frequencies up to $\sim 100 \mathrm{GHz}$, the event is dominated by non-thermal emission associated with hard $\mathrm{X}$-rays and high-energy gamma-rays, while at $345 \mathrm{GHz}$ it is consistent with optically thick thermal emission from chromospheric regions at $\sim 2 \times 10^{5} \mathrm{~K}$ (Trottet et al. 2008). The measured source size of the thermal source, $60^{\prime \prime}$, is again large (Lüthi et al. 2004a). These findings indicate that the observations of the radio continuum at centimeter-submillimeter wavelengths can serve as a powerful diagnostic tool to study the structure and the dynamics of the low solar atmosphere as well as energy transport during flares.

\subsection{Gyromagnetic radiation}

Gyromagnetic radiation is the electromagnetic radiation of an electron or positron gyrating under the Lorentz force in a magnetic field.

The fundamental frequency of the radiation is the electron cyclotron frequency $v_{\mathrm{c}}$ (= $2.8 \times 10^{6} \mathrm{~B} \mathrm{~Hz}$, with $B$ measured in gauss). At low energies the radiation will be restricted to this frequency and low harmonics. With increasing particle energy radiation at higher harmonics becomes more efficient and the radiation becomes more and more beamed in the direction of motion of the particle. Taking the Doppler shift due to the field-aligned motion of the particle into account, the emission frequency of an electron or positron with Lorentz factor $\gamma$ at the harmonic $s$ is

$$
2 \pi \nu=s \frac{2 \pi v_{\mathrm{c}}}{\gamma}+k_{\| v_{\|}}
$$


or

$$
v=s \frac{v_{\mathrm{c}}}{\gamma} \frac{1}{1-n \beta \cos \alpha \cos \theta} .
$$

Here $n$ is the refractive index of the plasma, $\beta$ the particle speed divided by the speed of light, $\alpha$ its pitch angle, and $\theta$ the angle between the viewing direction and the magnetic field.

\subsubsection{Incoherent gyrosynchrotron and synchrotron radiation of energetic electrons and positrons}

"Gyrosynchrotron" is the label used to describe gyromagnetic radiation of mildly relativistic electrons $(0<\beta \ll 1)$, while "synchrotron emission" designates the ultrarelativistic limit $(\gamma \gg 1, \beta \rightarrow 1)$. The difference is not in the physical mechanism, but in the approximations made in the calculation of the coefficients of emission and absorption. The spectrum is a series of spectral lines whose width increases and separation decreases with increasing Lorentz factor. Above some harmonic number the spectrum is virtually continuous. The integration over the pitch angle and energy distributions of a particle population, rather than a single particle, further smears individual harmonics.

The emission of a single particle covers a band whose width increases with increasing energy. A typical frequency for the maximum emission of an electron with Lorentz factor $\gamma$ and pitch angle $\alpha$ is, in the ultrarelativistic approximation (e.g., Pacholczyk 1970, Eq. (3.28)),

$$
v_{0}=\frac{3}{2} \gamma^{2} v_{\mathrm{c}} \sin \alpha,
$$

implying $s \propto \gamma^{2}$. This suggests that mildly relativistic electrons are most efficient emitters at low harmonics of the cyclotron frequency, as confirmed by numerical calculations.

While the emissivity of a single particle increases and its spectrum broadens with increasing energy, most particle distributions decrease with increasing energy. As a result the emissivity and absorption coefficient of, e.g., a power-law distribution of particles decrease with increasing frequency. Intensity spectra that rise at low frequencies can be produced by a low-energy cutoff of the radiating electrons in the $\mathrm{MeV}$ range, by self-absorption, free-free absorption in the ambient thermal plasma or at a plasma screen outside the radiation source, and by suppression of the gyromagnetic emission by the ambient medium. The action of the ambient medium is readily seen in Eq. (12), where the particle velocity $\beta$ is multiplied by the refractive index $n$, which is smaller than unity in the plasma. Clearly the action of the ambient plasma here appears as if the particle velocity were lowered, thus diminishing emission. This effect is called Razin suppression, and it operates on both the emissivity and absorption coefficient. Razin suppression occurs at frequencies above, but not too far from, the electron plasma frequency of the medium. A handy expression (in cgs units) for the frequency below which Razin suppression is important, again in the ultrarelativistic regime, is

$$
v_{R}=\frac{2}{3} \frac{v_{p e}^{2}}{v_{\mathrm{c}} \sin \alpha} \simeq 20 \frac{n_{e}}{B} \mathrm{~Hz}
$$


(e.g., Pacholczyk 1970, Eq. (4.10)). Razin suppression creates a very steep lowfrequency cutoff, which is not due to absorption, but to the suppression of energy transfer from the particles to electromagnetic waves in the vicinity of the plasma frequency.

The observed frequency spectra of microwave bursts statistically peak near $10 \mathrm{GHz}$, but the peak frequency increases with increasing peak flux density (Fürst 1971; Guidice and Castelli 1975; Nita et al. 2004), and can exceed $20 \mathrm{GHz}$ in major events (Correia et al. 1994; Ramaty et al. 1994). A peak frequency of $10 \mathrm{GHz}$, together with a typical harmonic number $s=5$, implies a typical magnetic field of $700 \mathrm{G}$ in the microwave source. This is of course nothing more than a rough estimate, because the spectral maximum of the gyrosynchrotron emission also depends on the column density of the radiating electrons, in line with the observed relationship between the frequency and flux density of the spectral maximum.

\subsubsection{Gyrosynchrotron radiation of non-thermal electrons and rising submillimeter spectra}

In the following we discuss the ranges of parameters that can produce an increasing spectrum with a peak frequency above $500 \mathrm{GHz}$. For simplicity we consider a single electron population with a power-law energy spectrum in a uniform magnetic field and a radio emitting source of projected diameter $d_{s}$ and depth $h_{s}$ along the line of sight. In addition to $B, d_{s}$, and $h_{s}$, even for such a simple model, there are a number of free parameters characterizing the ambient plasma (temperature $T$ and electron density $n_{e}$ ) and the instantaneous density of radio-emitting electrons above $20 \mathrm{keV}$, $n_{20}$, and the power-lax spectral index $\delta$. As observations at two frequencies are not sufficient to determine this ensemble of free parameters we take $n_{20} \approx 10^{37} \mathrm{~cm}^{-3}$ and $\delta=3$, values used in Trottet et al. (2008) for the SOL2003-10-28 event. We arbitrarily consider $h_{s}=1^{\prime \prime}$.

Figure 13 shows examples of gyrosynchrotron spectra which broadly agree with the range of flux densities observed at 212 and $405 \mathrm{GHz}$ at the maxima of THz events, indicated by the two vertical bars.

- The spectrum shown by the full line is obtained for $B=1500 \mathrm{G}$ and $d_{s}=1^{\prime \prime}$. The small size of the source is necessary to push the non-thermal number densities to very high values and thus contribute, in conjunction with the strong magnetic field, to creating a very high peak frequency, as shown in Fig. 4. With such parameters gyrosynchrotron self-absorption creates a spectrum that rises up to about $800 \mathrm{GHz}$. This interpretation was explored by Silva et al. (2007) to explain the SOL2003-11-02 flare observation by SST: they adopt a broken power-law electron energy distribution of slope 4.15 below $3 \mathrm{MeV}$ and 3.15 above containing $5 \times 10^{35}$ electrons above $50 \mathrm{keV}$ confined to a region $0.5^{\prime \prime}$ in size where the magnetic field strength is $4500 \mathrm{G}$. The parameters for the electron energy distribution are chosen to match the hard X-ray emission observed in this event. However, there is an upper limit to the possible values of magnetic field strength in the corona, set by the largest values seen in the photosphere ( $4000 \mathrm{G}$, e.g., Zirin and Wang 1993). Coronal field strengths of $2000 \mathrm{G}$ are commonly observed in large active regions by the Nobeyama Radioheliograph (NoRH) at $17 \mathrm{GHz}$ (Shibasaki et al. 1994), 


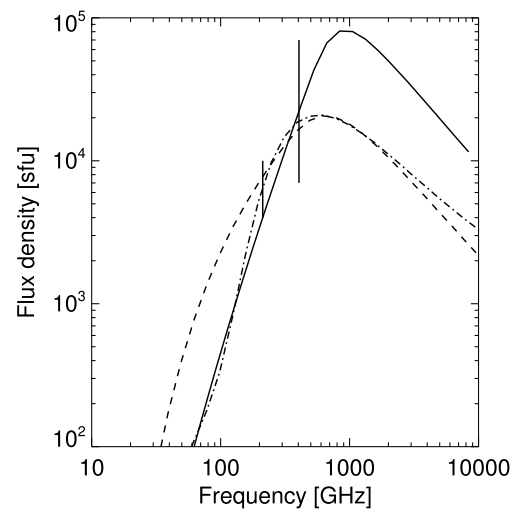

Fig. 13 Examples of gyrosynchrotron radio spectra for emission by electrons with a power-law energy distribution with a spectral index of 3 in a source of homogeneous magnetic field. The angle between the line of sight and the direction of the magnetic field is taken to be $45^{\circ}$. The full, dashed and dashed-dotted lines correspond, respectively, to models where the low-frequency part of the radio spectrum is shaped by self-absorption, free-free absorption and Razin suppression (see text for the parameters used). The vertical bars indicate the ranges of flux densities observed at 212 and $405 \mathrm{GHz}$ in $\mathrm{THz}$ flares

but field strengths in the corona have never been observed to reach $4000 \mathrm{G}$ (when they would be observed by NoRH at $34 \mathrm{GHz}$ ). This implies that the largest possible value of $v_{\mathrm{c}}$ in the corona is of order $8 \mathrm{GHz}(3000 \mathrm{G})$. While the self-absorbed spectrum in Fig. 13 gives a possible representation of the observations with a plausible magnetic field strength in the low corona, a persistent problem is the required nonthermal electron density. The high value of the density of non-thermal electrons in the radio emitting source $\left(n_{20}=2-4 \times 10^{13} \mathrm{~cm}^{-3}\right)$ required in order to achieve a high turnover frequency would be a significant fraction of the total electron density in the chromosphere, and unrealistically high for the flaring corona.

- The dashed line shows a spectrum where free-free absorption in the emitting source itself dominates below the spectral maximum. It has been obtained for $B=700 \mathrm{G}, d_{s}=10^{\prime \prime}, n_{e}=1.5 \times 10^{12} \mathrm{~cm}^{-3}$ and $T=10^{5} \mathrm{~K}$. In this case, $n_{20} \approx 3 \times 10^{11} \mathrm{~cm}^{-3} \approx 0.2 n_{e}$. The temperature implies a source in the transition region, so that the thermal electron density is moderate. It is, however, questionable if this localization is consistent with the required size $d_{s}$.

- The spectrum shown by the dashed-dotted line is shaped by Razin suppression below $220 \mathrm{GHz}$. The adopted parameters are $B=700 \mathrm{G}, d_{s}=10^{\prime \prime}, n_{e}=8 \times$ $10^{12} \mathrm{~cm}^{-3}$ and $T=5 \times 10^{6} \mathrm{~K}$. In this case $n_{20} \approx 3 \times 10^{11} \mathrm{~cm}^{-3} \approx 0.04 n_{e}$.

Although the above discussion is based on a specific set of parameters that is not uniquely defined, it indicates that self-absorption as well as free-free absorption may not be appropriate to account for increasing spectra in the $200-400 \mathrm{GHz}$ domain because extremely high densities of energetic electrons are needed. The estimates above constitute lower limits because free-free absorption by a plasma screen outside the radiating source was not considered (such an absorbing screen is more likely if the emitting source is low in the solar atmosphere). Moreover, the observations often show an almost linear correlation between the submillimeter emission and the hardX-ray peaks (see Sect. 3.2), whereas self-absorbed gyrosynchrotron, which depends 
on the product of the effective energy of the emitting electrons and the area of the optically thick source, is not expected to show such a strong correlation. Such a correlation is expected for free-free absorption of optically thin gyrosynchrotron emission, since the variation of the non-thermal electron density only affects the emissivity, and for Razin suppression, because the $200-400 \mathrm{GHz}$ emission is optically thin even though the spectrum rises with increasing frequency. For the parameters used above one needs $5 \times 10^{6} \mathrm{~K}<T<10^{7} \mathrm{~K}$ in order to avoid too much free-free absorption and too high a contribution to the hot soft X-ray source measured by GOES. But given the high temperature, the $\mathrm{THz}$ source must be located in the flaring corona, where the ambient density required for Razin suppression again appears rather high.

It is clear that the microwave component of the spectra in Fig. 13 is much weaker ( $<100 \mathrm{SFU}$ ) than that observed during THz bursts $\left(>10^{4} \mathrm{SFU}\right.$ around $15 \mathrm{GHz}$ ). This implies that the usual microwave emission arises from a separate region of weaker magnetic field. Silva et al. (2007) model the microwave emission of the SOL2003-1102 flare using the same electron spectrum as in the $\mathrm{THz}$ source, but with a microwave source that is 100 times larger and has a magnetic field $10 \times$ smaller.

In summary, gyrosynchrotron radiation from non-thermal electrons can in principle explain radio spectra rising through the mm-wave range, but at the expense of requiring extreme values for the non-thermal or ambient electron density, or both. It is not clear how such a large non-thermal population is accelerated, nor how it can be maintained for the duration of this event. Furthermore, the confinement of such an electron population at such a high energy density is unclear (the required confining magnetic field for our three examples are $7000 \mathrm{G}, 700 \mathrm{G}$, and $700 \mathrm{G}$, respectively, while for the parameters proposed by Silva et al. (2007) require a magnetic field strength of $5500 \mathrm{G}$ ). The way in which the flare maintains two separate energetic electron populations radiating intensely in microwaves and in the mm-wave range, but with a gap in between, while showing some similarity of time profiles that argue for a direct magnetic connection between the two sources, is also not easily understood. The numerical evaluations presented here and in the literature hence do not completely rule out the incoherent gyrosynchrotron mechanism based on nonthermal electrons (see also Melnikov et al. 2013), but they clearly motivate a search for alternatives.

\subsubsection{Synchrotron emission from relativistic positrons}

Gyrosynchrotron emission of positrons, instead of electrons, provides an attractive alternative because it might naturally explain the gap between the microwave spectrum and the $\mathrm{THz}$ spectrum, while maintaining a close link between the time histories of the radiating particle populations as revealed by common time structures. The impulsive phase emission of SOL2003-10-28 started simultaneously with the $\gamma$-ray emission above $60 \mathrm{MeV}$ (Fig. 8). This high-energy $\gamma$-ray continuum is predominantly produced by $\Pi^{0}$ decay photons and by bremsstrahlung from relativistic positrons from the decay of $\Pi^{+}$secondaries, bremsstrahlung from relativistic electrons from the decay of $\Pi^{-}$being negligible (e.g., Murphy et al. 1987; Vilmer et al. 2003). Moreover, the source at $210 \mathrm{GHz}$ was found to be compact (Lüthi et al. 2004a) and co-spatial with one of the $2.2 \mathrm{MeV}$ line sources (Trottet et al. 2008), i.e. with one 
of the ion interaction regions observed by RHESSI Hurford et al. (2006). These facts suggest that the $\mathrm{THz}$ emission may be produced by relativistic positrons. The possibility that positrons contribute to the microwave spectrum by synchrotron emission was first pointed out by Lingenfelter and Ramaty (1967). For magnetic fields of the order of $1000 \mathrm{G}$ the peak frequency of the synchrotron spectrum from positrons (or electrons) with energies typical of pion decay products falls in the sub-mm- or mmwave range.

The resulting synchrotron emission is thought to be optically thin, but the peculiar energy spectrum of the positrons, which increases up to $\sim 10 \mathrm{MeV}$ as roughly the square of the positron energy, could create a synchrotron spectrum with a positive slope. The broad spectrum of the emission of individual positrons above about $1 \mathrm{MeV}$ makes this slope rather shallow: the low frequency part of the synchrotron spectrum increases as $v^{1 / 3}$ (e.g., Pacholczyk 1970, Eq. 6.30). This is inconsistent with the observed slope $(\geq 1)$ in the $200-400 \mathrm{GHz}$ domain. However, a combination of freefree absorption and Razin suppression is capable of producing the required spectral shape (cf. Trottet et al. 2008; Fleishman and Kontar 2010). As discussed above, this requires high plasma densities $\left(\geq 10^{12} \mathrm{~cm}^{-3}\right)$ and plasma temperatures in the $0.5-1 \times$ $10^{6} \mathrm{~K}$ range. Nevertheless, to match the observed flux densities in the $200-400 \mathrm{GHz}$ range, it seems that this mechanism requires more relativistic positrons than created even in the most powerful $\gamma$-ray flares. The instantaneous number of positrons needed is estimated to be of the order of a few times $10^{31}$ to get flux densities of $\sim 10^{4} \mathrm{SFU}$. As the number of positrons produced by $>200 \mathrm{MeV}$ protons is in the range $10^{-1}$ $10^{-2}$ per proton (cf. Fig. 3 in Lingenfelter and Ramaty 1967), a few times $10^{32}-10^{33}$ protons above $200 \mathrm{MeV}$ are needed to account for the radio emission. This is at least an order of magnitude larger than the range of values derived from the largest fluxes of pion-decay photons observed so far (cf. Vilmer et al. 2003). Although incoherent synchrotron emission from relativistic positrons seems to be ruled out, this does not necessarily imply that positrons do not play a major role in the production of the $\mathrm{THz}$ component through synchrotron maser or Vavilov-Cherenkov radiation (see the discussion below).

\subsubsection{Gyrosynchrotron radiation from thermal electrons}

Gyrosynchrotron radiation by electrons is not restricted to non-thermal populations with a power law energy spectrum, but can also be produced by a very hot thermal population. Since the simultaneous presence of very hot thermal and non-thermal electron populations is well established in solar flares, the two populations are a possible means to explain the distinct microwave and $\mathrm{THz}$ spectral components. Thermal electron populations can be created directly during the flare energy release or through the impact of energetic particles onto the chromospheric footpoints, which causes the ionization and heating of the plasma, and flow upwards into the flaring loops. The plasma filling these loops has a temperature of order 20 million degrees, as revealed by extremely hot EUV lines and densities of $10^{12} \mathrm{~cm}^{-3}$ (e.g., Doschek et al. 1996). In order to produce optically thick gyrosynchrotron emission in the sub-THz range, however, much hotter electrons together with very strong coronal magnetic fields are required: at a temperature of about $T_{m} \approx 10^{8} \mathrm{~K}$, electrons gyro-radiating in a $5000 \mathrm{G}$ 


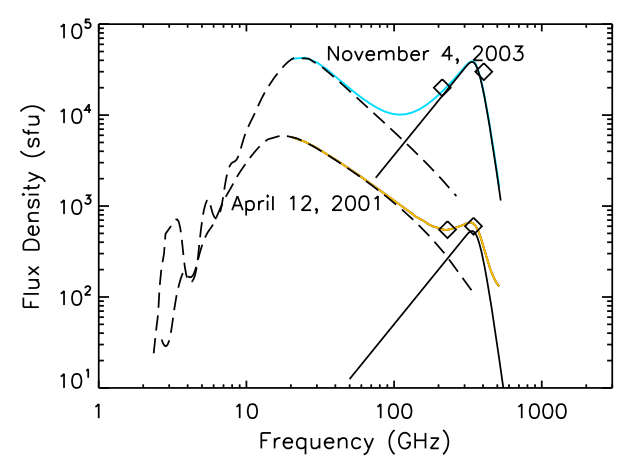

Fig. 14 Spectral fits using gyrosynchrotron emission from non-thermal electrons plus thermal synchrotron. The blue curve represents the solution for the event SOL2003-11-04T19:44, with the following parameters for the thermal solution: $T_{e}=2.8 \times 10^{8} \mathrm{~K}, n_{e}=8 \times 10^{11} \mathrm{~cm}^{-3}$, diameter $=15^{\prime \prime}$, length $=10^{9} \mathrm{~cm}$. The yellow curve is the solution for the SOL2001-04-12T10:28 event. The parameters of the thermal source are: $T_{e}=2.8 \times 10^{8} \mathrm{~K}, n_{e}=8 \times 10^{11} \mathrm{~cm}^{-3}$, diameter $=1.8^{\prime \prime}$, length $=10^{9} \mathrm{~cm}$. The magnetic field $B=5200 \mathrm{G}$ for both solutions

magnetic field will have a radio spectrum peaking at around a few hundred GHz. Costa et al. (2013) argue that this can account for most of the flux detected during the observations.

Two examples of a thermal synchrotron spectrum are shown in Fig. 14. Either would be in conflict with the GOES soft X-ray photometry because of the high temperatures and emission measures (e.g., Garcia 1994). Furthermore, the magnetic field strength used in the models of Fig. 14 is beyond acceptable limits, especially with a scale size of $10^{9} \mathrm{~cm}$. Semi-empirical formulas of gyrosynchrotron radiation from a thermal plasma (cf. Dulk 1985) show that the peak frequency depends essentially on the magnetic field strength and the temperature, and very weakly on the column density of the radiating electrons (see Fig. 4). There seems to be little room for removing the requirement of an extremely high magnetic field by accommodating other parameters. The thermal gyrosynchrotron model hence does not work; the $50 \mathrm{keV}$ HXR flux predicted by the two models shown in Fig. 14 would be $3 \times 10^{5}$ and $4 \times 10^{3} \mathrm{ph} /\left(\mathrm{cm}^{2} \mathrm{~s} \mathrm{keV}\right)$, respectively, each well in excess of what is observed (largest flares have peak flux at $50 \mathrm{keV}$ of a several times $\left.10 \mathrm{ph} /\left(\mathrm{cm}^{2} \mathrm{~s} \mathrm{keV}\right)\right)$.

\subsection{Coherent emission mechanisms}

Coherent emission mechanisms are known to play an important role in the solar corona at frequencies of a few $\mathrm{GHz}$ (Slottje 1978) and below. They are rarely observed at higher frequencies: the highest-frequency examples are perhaps those reported by Benz et al. (1992), who observed millisecond spike bursts at frequencies up to $8 \mathrm{GHz}$, and by Staehli et al. (1987), who report some evidence of harmonic structure up to about $12 \mathrm{GHz}$. The latter note that such signatures are rare. Coherent radio bursts are usually readily identifiable via their extreme brightness temperatures, spectral signatures, rapid fluctuations, and/or polarization properties. While coherent radiation mechanisms may be relevant at millimeter or submillimeter wavelengths in principle, there are no compelling examples that require a coherent mechanism to 
account for the observations. In the case of the $\mathrm{THz}$ component, however, coherent mechanisms cannot be definitely excluded either. Several coherent mechanisms are therefore listed below and could be relevant in principle.

\subsubsection{Cherenkov radiation}

Fleishman and Kontar (2010) introduced a novel idea for the origin of the submillimeter radiation: Cherenkov radiation of fast electrons with velocities exceeding the speed of light in the local medium. Ultrarelativistic electrons and positrons in cosmic-ray showers produce such radiation in air and it has long been used as a standard tool for studying cosmic rays (e.g. Jelley 1955). The time development of such emission would reflect the acceleration and transport of flare energetic electrons (as well as any variations in the refractive index of the medium due to composition changes) so it could easily fluctuate very rapidly. The intensity and spectral form of Cherenkov emission depend crucially on the dielectric properties of the solar atmosphere. Cherenkov radiation is perhaps unfamiliar to many so we discuss these features briefly to recall its main properties.

The rate $\mathrm{d} I / \mathrm{d} v\left(\mathrm{erg} \mathrm{s}^{-1} \mathrm{~Hz}^{-1}\right)$ of Cherenkov radiation at frequency $v$ by an electron of speed $\beta c$ moving in a medium of dielectric permittivity $\epsilon(v)$ (equal to the square of the refractive index) is given by the Tamm-Frank formula (e.g. Jackson 1962):

$$
\frac{\mathrm{d} I}{\mathrm{~d} \nu}=\frac{4 \pi^{2} e^{2}}{c} \nu\left(1-\frac{1}{\beta^{2} \epsilon(\nu)}\right) .
$$

Cherenkov radiation is effective if $\beta^{2} \epsilon(\nu)>1$. To calculate the total Cherenkov flux at some frequency $v$, we multiply Eq. (15) by the energy distribution of electrons and integrate over all energies such that $\beta>1 / \sqrt{\epsilon(v)}$.

If $\epsilon$ depends only weakly on $\nu$, then we have roughly

$$
\frac{\mathrm{d} I}{\mathrm{~d} v} \propto v
$$

i.e. a continuous spectrum that increases with frequency, but less rapidly than is actually observed. If Cherenkov radiation is to account for flare submillimeter, $\epsilon(\nu)$ must have a significant $v$-dependence so that the number of radiatively effective electrons increases with $v$.

Cherenkov emission emerges in a cone around the direction of motion of the electron, of half-angle $\theta$ given by

$$
\cos \theta=\frac{1}{\beta \sqrt{\epsilon(\nu)}} .
$$

Thus the radiation from a continuous distribution of electron energies will occupy the whole of the inside of a cone of half-angle $\cos ^{-1}(1 / \sqrt{\epsilon(v)})$.

A spectral line at frequency $\nu_{0}$ adds a contribution to the dielectric permittivity that behaves as (e.g. Heitler 1954)

$$
\sim \frac{1}{\left(v-v_{0}\right)^{2}+i \Gamma},
$$

where $\Gamma$ is the decay constant of the transition. 

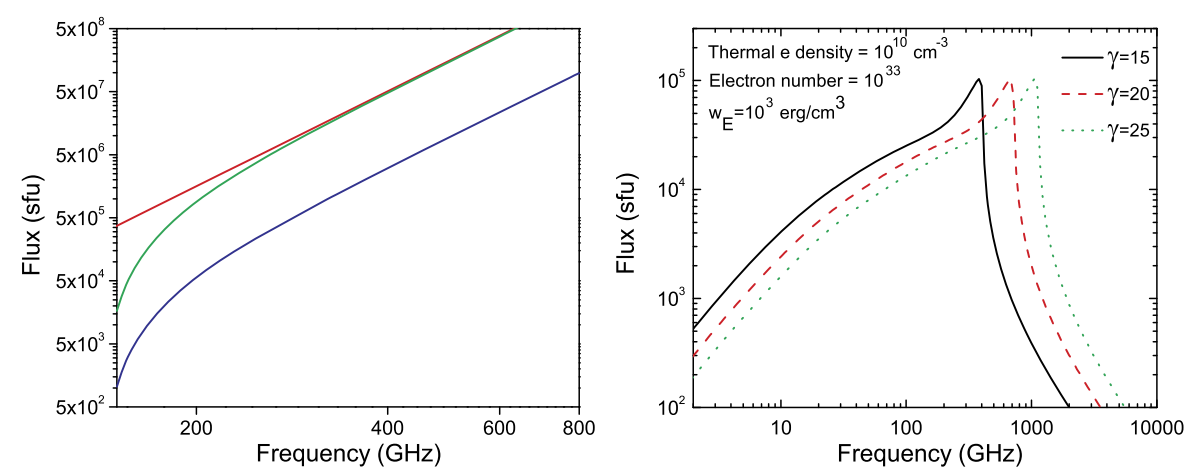

Fig. 15 Left: model spectra of the Cherenkov mechanism (power-law spectra, with red and green lines showing two different parameter choices; red line, ignoring effects near the plasma frequency); right: diffusive radiation in Langmuir waves, showing the dependence on electron energy $\gamma$ for a fixed energy density in Langmuir waves (Fleishman and Kontar 2010)

Fleishman and Kontar (2010) suppose that very many (possibly overlapping) atomic and molecular transitions produce a net dielectric permittivity that may be modeled as

$$
\epsilon(v)=1-\frac{v_{p}^{2}}{v^{2}}+\frac{v^{2}}{v_{0}^{2}} .
$$

In principle a complete line list including wavelengths and oscillator strengths together with statistical equilibrium calculations could give $\epsilon(v)$ exactly: however, Fleishman and Kontar (2010) do not make such a calculation and merely speculate that it would produce a result of the form (16). Equation (16) does allow some parametric discussion in the absence of this challenging calculation, but the key parameter $\nu_{0}$ is completely unknown at present. Using this $\epsilon(\nu)$ in Eq. (15) with a speed distribution $\propto \beta^{-b}$, and adopting values of $v_{0}$ in the THz range, Fleishman and Kontar find a spectrum that can increase with $v$ (Fig. 15, left panel).

The above description of the Cherenkov physics is ad hoc and its proposers do not provide concrete estimates based on experimental results. We can therefore consider cosmic-ray air showers, which develop in the Earth's atmosphere, and for which coherent $\mathrm{THz}$ emission has not apparently been reported. In air the refractivity $N=(n-1) \times 10^{-6}$ is of order 300 , where $n$ is the refractive index. Empirically the refractivity at microwave frequencies is not very different (e.g. Bean and Dutton 1961). For solar plasma we can crudely estimate the refractivity by using air via scaling to the density of oxygen, which gives a factor of roughly $10^{-4}$ and a refractivity of about 0.3 . In a sense this is an upper limit since air is composed almost entirely of molecules and has a rich mm-wave spectrum due to water vapor, for example. For this refractivity, Eq. (15) shows the relevant electron energy to be about $600 \mathrm{MeV}$ and for a simple power-law extrapolation of the electron spectrum as $E^{-3}$, one finds $2 \times 10^{28}$ electrons and an ample $300 \mathrm{GHz}$ flux. This electron energy is well above the value $E_{\text {roll }} \approx 40 \mathrm{MeV}$ value of the high-energy cutoff estimated by Vilmer et al. (2003), so it is not clear if it is realistic. We conclude that the Cherenkov mechanism implies extreme parameters, but should be investigated further. 
A further possibility is Cherenkov radiation from secondary positrons and electrons produced via the decay of charged pions in $\mathrm{p}-\mathrm{p}$ interactions (Murphy et al. 1987). These naturally have an energy distribution peaking about $150 \mathrm{MeV}$, making them the most relativistic particles present in flares (in terms of Lorentz $\gamma$ ), and thus prime candidates to radiate via the Cherenkov mechanism. A large flare might involve as many as $\sim 10^{31}$ protons above $300 \mathrm{MeV}$ (e.g. Vilmer et al. 2003), and thus $\sim 10^{29}$ positrons (e.g. Murphy et al. 1987). If we suppose that all positrons are so energetic that they satisfy $\beta \sqrt{\epsilon(\nu)}>1$, Eq. (15) implies a flux of $<4 \times 10^{4}$ SFU at $400 \mathrm{GHz}$ (the upper limit applying in the case $\sqrt{\epsilon} \gg 1$ ). This estimate unrealistically assumes that the positrons remain at their original energies in the source for the lifetime of the event. A more detailed calculation for individual events may be merited but this would have to be supplemented by a more realistic consideration of the atmospheric dielectric properties.

\subsubsection{Diffusive radiation in Langmuir waves}

In this emission mechanism (e.g. Fleishman and Toptygin 2007a, 2007b) flareaccelerated electrons at relativistic energies are assumed to encounter a region with turbulent waves (e.g. in a flare loop) (e.g. Tsytovich et al. 1975). The electric fields of the turbulent wave then act as a random Lorentz force on the relativistic electrons creating electromagnetic emission termed "diffusive radiation" (the term diffusive is used to describe the random walk that electrons undergo in this process). For longwavelength Langmuir waves, this process can produce a positive slope (power law index up to 2) in the submillimeter range with the peak frequency given by $2 v_{p} \gamma^{2}$, where $v_{p}$ is the plasma frequency (Fleishman and Kontar 2010). To have an increasing spectrum up to $400 \mathrm{GHz}$, the ambient density in the turbulent region therefore must by $n_{e}>4 \times 10^{14} \gamma^{-4} \mathrm{~cm}^{-3}$. For electrons with $\gamma=10$, this gives densities above $4 \times 10^{10} \mathrm{~cm}^{-3}$, values that are typical for flare loops. The electron population (number of electrons with energy $\gamma$ ) and the energy density in Langmuir waves are the parameters required to calculate the radio flux. For $10^{33}$ electrons with $\gamma=15$ (a large number) and energy density of $10^{3} \mathrm{erg} \mathrm{cm}^{-3}$, fluxes at $400 \mathrm{GHz}$ of $4 \times 10^{4}$ SFU could be produced (Fleishman and Kontar 2010); see Fig. 15 (right panel). This are values similar to seen for the largest events. However, the required energy density of Langmuir waves is large, corresponding to the energy density of a magnetic field of about $170 \mathrm{G}$, and the origin of these waves is not known. It is thus difficult to make quantitative statements. Nevertheless, the characteristic spectrum (Fig. 15, right panel) with a sharp decrease after the peak frequency, would provide an excellent diagnostic for future observations to distinguish this process from some other mechanism.

\subsubsection{Coherent-bunch synchrotron radiation}

This model addresses the presence of two distinct components in the radio spectrum by attributing the submillimeter component to normal synchrotron emission, as discussed above, while using emission from coherent bunches to explain the microwave component (Kaufmann and Raulin 2006). Coherent bunching radiation works as follows: if $\mathrm{N}$ electrons move together within a volume of dimension much smaller than a 
wavelength, then instead of radiating as $\mathrm{N}$ independent charged particles, they emit as if they were a single electron of charge $\mathrm{N} e$. The radiation from such a coherent group of electrons is then proportional to $\mathrm{N}^{2}$ rather than $\mathrm{N}$. This phenomenon is common in laboratory devices such as particle colliders and free-electron lasers. The idea for submillimeter bursts is that all the radio emission is produced by a single population of highly relativistic electrons, but that the electrons are confined to bunches of dimension less than $1 \mathrm{~cm}$, and so radiate coherently at longer wavelengths but incoherently at shorter wavelengths. This effect elevates the flux at longer wavelengths and makes it appear to be a separate component with a spectral peak in the microwave range.

The spectrum will appear to contain two components as long as the electrons remain confined to bunches smaller than $1 \mathrm{~cm}$. However, any spread in electron velocities will quickly disperse the bunches and terminate the coherent emission in the microwave range. For this reason, this model requires the electrons to be highly relativistic (in order to produce synchrotron emission at submillimeter wavelengths) and essentially mono-energetic (to prevent bunch dispersion; highly relativistic electrons will all have velocities close to $c$ ). In the case of synchrotron emission where the gyroradius is of the same order as the wavelength of radiation, the electrons must gyrate together bunched in phase around the gyro-orbit. Since the same electrons produce the radiation at all radio wavelengths, the microwave and submillimeter components should behave in a tightly correlated manner, but the ratio of the two components may vary. The microwave component would presumably be more highly variable as bunches are created and eventually disperse due to the spread in velocities.

Possible mechanisms for generating bunches are not well established. Klopf et al. (2010) note that such bunches are deliberately created in free-electron lasers using "undulators" (periodic magnetic structures) in order to create powerful radiation sources. There is also a self-bunching mechanism involving the ponderomotive force of the bunched radiation. Unless such self-bunching is a robust and universal process, it seems likely that the bunches must be generated intrinsically by the acceleration mechanism: such small groups of electrons do not seem to be a natural consequence of current candidate acceleration mechanisms.

Note that in this model the coherent bunching radiation is used to explain the more common component of the radio spectrum (the microwave-peaked component). Since this component does not seem to differ between flares with and without the submillimeter component, one is forced to conclude that the coherent bunching mechanism is always operating in flares to produce the microwave emission. This seems unlikely in view of the extreme conditions needed to maintain the bunches against velocity dispersion, and the fact that the incoherent gyrosynchrotron emission mechanism comfortably explains the properties of most observed microwave bursts, as well as their excellent correlation with hard X-ray emission.

Another issue for this mechanism is that in order for the submillimeter component to have a rising spectrum, it must be optically thick, and this implies that the lowerfrequency microwave component is also in the optically thick regime. The effect of bunching in the optically thick limit seems not to have been addressed theoretically, since it is not relevant to the collider and free-electron laser circumstances. A simple argument is as follows: by Kirchoff's law, the brightness temperature of the emission in the optically thick limit simply corresponds to the effective energy of the radiating 
particles, and the effective energy of a particle consisting of a coherent bunch of $\mathrm{N}$ electrons moving together is $\mathrm{N}$ times the energy of a single electron. A more careful inspection of the emissivity and absorption coefficients for synchrotron emission confirms this argument. Thus if this mechanism works, it can make the microwave emission up to $\mathrm{N}$ times brighter than the extension of the submillimeter spectrum down to microwave frequencies. However, this multiplication factor also implies that extreme brightness temperatures, well in excess of the $10^{9}-10^{10} \mathrm{~K}$ values implied by electron energies of order $100 \mathrm{keV}$, should be measured in the microwave component. Current microwave imaging measurements seldom reach brightness temperatures of $10^{9} \mathrm{~K}$ at a spatial resolution of order $5-10^{\prime \prime}$, so much higher intrinsic brightness temperatures require very small (subarcsecond) microwave sources in the optically thick limit.

\subsubsection{Synchrotron maser}

The electron cyclotron maser (ECM) involving mildly relativistic electrons has been much invoked in astrophysics (e.g. Wu 1985; Treumann 2006). Operating near the local gyrofrequency $\Omega_{e}$, ECM cannot be important in the submillimeter range even for the strongest magnetic fields found on the Sun. The association of submillimeter radiation with $\gamma$-rays raises the possibility of maser operation involving relativistic electrons, or possibly positrons, however. This would constitute a synchrotron maser (McCray 1966; Zheleznyakov 1967) potentially operating at much higher harmonics of the gyrofrequency, and in the regime where harmonics merge to form a continuum. Maser action by electrons in the MeV energy range has been discussed in Wu (1985) and Louarn et al. (1987). Direct electromagnetic radiation can occur at larger values of $v_{p} / \Omega_{e}$ than for the mildly relativistic ECM, mostly at $2 \Omega_{e}$ where it can escape more easily, without re-absorption at an overlying harmonic layer.

One essential ingredient is a region of velocity space where $\partial f / \partial p>0$, a situation which would develop naturally as electrons of lower energies stop higher in the atmosphere (e.g., Emslie and Smith 1984); or which would automatically be met for the electron and positron distributions resulting from $\mathrm{p}$-p collisions and pion decay (Murphy et al. 1987). The high energies involved in the latter case and the naturally occurring positive slope of the energy spectrum of electrons and positrons produced during these nuclear reactions make this a potentially appealing mechanism. It remains unclear whether enough particles can be present in the same region of the atmosphere. A detailed calculation of positron production and transport would have to be combined with growth rate calculations in the relativistic regime; the expressions of Cawthorne (1985) and Crusius-Waetzel (1991), assuming isotropic distributions and randomly directed magnetic fields, might serve as first estimates.

\subsubsection{Coherent plasma radiation}

Coherent plasma radiation from suprathermal electrons produced at the plasma frequency of the ambient medium is a well established emission mechanism at frequencies below $\sim 10 \mathrm{GHz}$ (Bastian et al. 1998). The lack of plasma emission at higher frequencies is generally attributed to the rapid increase of free-free absorption with 
frequency: since free-free opacity varies as $n_{\mathrm{e}}^{2} / T^{1.5}$, where $n_{\mathrm{e}}$ is the ambient electron density, and the plasma frequency $\nu_{\mathrm{p}}$ varies as $n_{\mathrm{e}}^{0.5}$, the free-free opacity at the plasma frequency rises roughly as $v_{\mathrm{p}}^{4}$. (In practice the absorption depends on the density gradient as the electromagnetic wave propagates away from the plasma frequency layer; see Benz 1993, Sect. 11.2). If the temperature of the medium is very high, this can offset the effect of increasing frequency, but to produce plasma emission in the $\mathrm{THz}$ range, densities of the order of $10^{15} \mathrm{~cm}^{-3}$ are required, and such densities are not reached in models of the quiet-Sun atmosphere until one drops below the $\tau=1$ layer in the photosphere where the temperature is less than $10000 \mathrm{~K}$ (e.g., Fontenla et al. 2007). Particle-in-cell (PIC) simulations of relativistic $(\gamma \approx 5)$ electron beams (Sakai et al. 2006) and proton beams with velocities of $\sim_{c} / 3$ (Sakai and Nagasugi 2007b) show that beams at high energies can indeed penetrate deep enough to reach such densities. Furthermore, plasma radiation in the mm-wave range with a higher flux at $400 \mathrm{GHz}$ than at $200 \mathrm{GHz}$ can be produced for relativistic electron beams as well as by proton beams. However, the problem of free-free absorption appears to be insurmountable: from Benz (1993), Eq. (11.2.5), a density scale height as small as $1 \mathrm{~km}$ still results in a free-free absorption optical depth of order $10^{7}$ for fundamental plasma emission at $400 \mathrm{GHz}$.

\subsection{Compton scattering}

High-energy electrons can Compton-scatter low-energy photons, for example converting gyrosynchrotron emission from long wavelengths up to $\mathrm{THz}$ frequencies. This is in principle interesting because at $10 \mathrm{GHz}$ one may have large optical depth and large photon densities. In the relativistic limit, the mean upscattering is given by the factor 4/3 $\gamma^{2}$ (e.g., Rybicki and Lightman 1985), which implies that a THz conversion from $10 \mathrm{GHz}$ photons requires a relativistic gamma of about 10 , or an electron energy of about $5 \mathrm{MeV}$. This does not sound improbable, but there are convincing arguments (e.g., Fleishman and Kontar 2010) that the photon energy density will always be much less than the magnetic energy density. This means that Compton scattering cannot compete with gyrosynchrotron emission (Rybicki and Lightman 1985).

\section{Future observations}

The spectral domain that is the subject of this review, basically the three decades between wavelengths of $1 \mu \mathrm{m}$ and $1 \mathrm{~mm}$, essentially remains virgin territory for solar flare research. The THz events that we have glimpsed hint strongly that new and interesting insights await better observations. A large part of the $\mathrm{THz}$ spectrum has not yet been covered by solar observations. However, rapidly evolving detector technology in the far infrared range makes this possible in the future.

Existing radio telescopes also have interesting capabilities. Among these we could name the 15-m James Clerk Maxwell telescope (Lindsey et al. 1990), the 10.4-m Caltech Submillimeter Observatory, the CARMA array, and the stratospheric observatory SOFIA. SST is still the only instrument to regularly observe the Sun at frequencies above $200 \mathrm{GHz}$. Its sensitivity was enhanced in 2006 with the upgrade 
Table 2 Summary of mechanisms that can produce positive $\mathrm{THz}$ slopes

\begin{tabular}{|c|c|c|c|}
\hline Mechanism & Arguments for & Arguments against & Ref. \\
\hline Free-free & Known mechanism & $\begin{array}{l}\text { Conflict with SXR for impulsive } \\
\text { phase }\end{array}$ & $\begin{array}{l}(1),(3), \\
(9)\end{array}$ \\
\hline $\begin{array}{l}\text { Synchrotron } \\
\text { (electrons) }\end{array}$ & Known mechanism & Extreme parameters required & $\begin{array}{l}(2),(3), \\
(9)\end{array}$ \\
\hline $\begin{array}{l}\text { Synchrotron } \\
\text { (positrons) }\end{array}$ & $\begin{array}{l}\text { Known mechanism } \\
\text { Correlation with } \gamma \text {-rays }\end{array}$ & $\begin{array}{l}\text { Extreme parameters required } \\
\text { Conflicts with } \gamma \text {-ray intensity }\end{array}$ & $(2),(3)$ \\
\hline $\begin{array}{l}\text { Synchrotron } \\
\text { (thermal) }\end{array}$ & Known mechanism & $\begin{array}{l}\text { Extreme parameters required } \\
\text { Conflict with HXR }\end{array}$ & (7) \\
\hline Cherenkov & Correlation with $\gamma$-rays & $\begin{array}{l}\text { Dielectric properties unknown } \\
\text { Highly relativistic particles needed } \\
\text { Inadequately studied }\end{array}$ & (4) \\
\hline Microbunching & Correlation with $\gamma$-rays & $\begin{array}{l}\text { THz from synchrotron } \\
\text { Bunching mechanism unknown } \\
\text { Coronal } \mu \text { waves unclear }\end{array}$ & (5) \\
\hline Plasma emission & $\begin{array}{l}\text { Correlation with } \gamma \text {-rays } \\
\text { High luminosity }\end{array}$ & Free-free absorption & (6) \\
\hline Diffusive radiation & Correlation with $\gamma$-rays & $\begin{array}{l}\text { Origin of Langmuir waves } \\
\text { Extreme parameters required }\end{array}$ & (4) \\
\hline Synchrotron maser & Correlation with $\gamma$-rays & $\begin{array}{l}\text { Extreme parameters required } \\
\text { Inadequately studied }\end{array}$ & (8) \\
\hline Inverse Compton & Correlation with $\gamma$-rays & Insufficient ambient photons & (4) \\
\hline
\end{tabular}

(1) Bastian et al. (1998); (2) Silva et al. (2007); (3) Trottet et al. (2008); (4) Fleishman and Kontar (2010); (5) Kaufmann and Raulin (2006); (6) Sakai and Nagasugi (2007a); (7) Costa et al. (2013); (8) This paper; (9) Kaufmann et al. (2009)

of the receivers at $212 \mathrm{GHz}$ reducing the system temperature and increasing their waveband. New facilities are being installed at the SST site to increase the frequency coverage. A small mid-IR camera in the $8-14 \mu \mathrm{m}$ band with a high image cadence is operating at CASLEO on a daily basis. Furthermore, full-Sun polarimeters at 45 and $90 \mathrm{GHz}$ are currently being installed (Polarization Emission of Millimeter Activity at the Sun (POEMAS), Valio et al. 2013). Their goal is to improve the characterization the intermediate region between the normal microwave gyrosynchrotron emission and the $\mathrm{THz}$ component. The former Bumishus patrol telescopes of the Bern University, now installed in Finland, operating between 1 and $50 \mathrm{GHz}$, will also be important to observe this intermediate region. A recently approved new submillimeter telescope that will be in operation by the fall of 2014, is the Large Latin American Millimeter Array (LLAMA, Arnal et al. 2011). While this 12-m single dish telescope is not solar-dedicated, it will occasionally provide high sensitivity observations of solar flares. Finally, a THz balloon-borne telescope, the Solar- $T$, is being developed to fly on a balloon. No imaging information will be available, but Solar-T will produce light curves at 3 and $7 \mathrm{THz}$ with a sensitivity $\sim 100$ s.f.u. The first science flight is expected to occur in 2013.

The DESIR project (Detection of Eruptive Solar Infra Red emission) was conceived for a microsatellite payload (e.g. Matthews et al. 2012). DESIR aims at photometric observations at two wavelengths with microbolometers. Use of a modest-size 
telescope (17 cm diameter) allows one to localize the emitting sources. DESIR was conceived as a pioneering instrument to open the far-infrared window on solar flares, but remains in the planning stage at present.

The Atacama Large Millimeter Array (ALMA) (see, e.g., Loukitcheva et al. 2008) offers the combination of excellent image quality and high spatial resolution (arcsecond) simultaneously for the first time. ALMA will be capable of spatial resolution rivalling the best solar telescopes at any wavelength. However, the limited field-ofview of ALMA ( $21^{\prime \prime}$ at $300 \mathrm{GHz}$ ) only covers a fraction of an active region reducing the chance of catching a flare significantly. Even for the case where ALMA is pointing at the flare location, the field-of-view is too small to contain all $\mathrm{THz}$ sources. Several options to improve flare observations with ALMA are available; two of them are mentioned here. ALMA could be split into sub-arrays to cover a larger area, at the cost of lower sensitivity and spatial resolution. To further enhance the chance of catching a flare, ALMA could be operated not as an interferometer, but in a single-dish mode. Each antenna is pointed at a slightly different location within the active region forming a mosaic pattern that covers the entire active region. Imaging information (at a much reduced level) would then be obtained in a similar fashion as currently been done with the multi-beam technique discussed in this review. A further key to the use of ALMA for solar observations lies in the implementation of an attenuator system, which will improve by a factor of 20 the basic system limit at about 5,000 K. This will definitely permit observations of the quiet Sun, active regions, and some flares. Saturation would occur for major $\mathrm{THz}$ events of the few thus far observed, but the enormous gain in sensitivity will certainly result in flare observations. Comparisons with hard X-ray sources, observed for example by RHESSI or by the STIX instrument on Solar Orbiter (Benz et al. 2012)), would be of primary interest. We eagerly look forward to learning how these new observations will relate to the observations and interpretations discussed in this review.

\section{Conclusions}

Solar flares generally have been detected at almost all wavelengths, but the three decades of wavelength in the far-IR/mm-wave spectral range have remained surprisingly ill-studied. This gap in our knowledge conceals unique important physics relating to high-energy particles and to the structure of the flaring atmosphere; only within the last solar cycle has a dedicated facility become (the SST at El Leoncito) become available. In this review we have critically assessed the observational material in the sub-THz band, corresponding to $0.1-0.2 \mathrm{~mm}$ wavelengths, most of which has come from a few events observed by the SST. Extremely valuable supplementary material also was obtained at the KOSMA instrument on Gornergrat, which is no longer active. There is virtually no observational material in the far-IR range yet.

The sub-THz observations of solar flares have now suggested two phenomena worthy of attention: a tendency towards pulsations, and an unexpected upward spectral break of the non-thermal radio spectrum that we have termed the "THz" component, with the clear implication that it may contain novel physics. Our main emphasis in this review has been to make a critical assessment of this $\mathrm{THz}$ component, and to give 
an overview of the several possible explanations. We deem that the pulsations may or may not have a particular connection to the $\mathrm{THz}$ band, and have mainly dealt here with the spectral signatures.

Our critical review has provided clear evidence that spectra with positive slopes extending towards the $\mathrm{THz}$ region do occur in major flares. Besides this new, but unexplained component, the observations also detect the optically thin low-temperature (a few $10^{6} \mathrm{~K}$ ) tail of the hot coronal sources readily observable in soft X-rays. In some events, there may also be an optically thick multi-temperature component at millimeter to submillimeter wavelengths with an increasing spectrum with frequency and with a few times the soft X-ray emission measure, but obviously arises from the chromospheric regions with temperatures in the $10^{3}$ to $10^{5} \mathrm{~K}$ range. There may indeed be more than one explanation, since some evidence for positive slopes exists in the pre-impulsive, impulsive, and even post-impulsive phases of a flare. In all three phases we find that non-thermal mechanisms must play a role, and the associations with hard X-rays and $\gamma$-rays suggest several interesting interpretations involving the high-energy particles fundamental to flare physics.

Acknowledgements The authors thank the International Space Science Institute (ISSI), Bern, Switzerland, for supporting the preparation of this review. We also would like to thank the anonymous referee for his comments, which helped to improve our review.

\section{References}

Akabane K, Nakajima H, Ohki K, Moriyama F, Miyaji T (1973) Sol Phys 33:431

Arnal EM, Morras R, Dubner GM, et al (2011) Bol Asoc Argentina Astron Plata Argentina 54:435

Bastian TS, Benz AO, Gary DE (1998) Annu Rev Astron Astrophys 36:131

Bean BR, Dutton EJ (1961) J Geophys Res 66:3717

Benz AO (1993) Plasma astrophysics: kinetic processes in solar and stellar coronae. Astrophysics and space science library, Vol. 184, Kluwer Academic, Dordrecht

Benz AO, Su H, Magun A, Stehling W (1992) Astron Astrophys Suppl Ser 93:539

Benz AO, Krucker S, Hurford GJ, et al (2012) Proc SPIE, 8443

Cawthorne TV (1985) Mon Not R Astron Soc 216:795

Cogdell JR (1972) Sol Phys 22:147

Correia E, Kaufmann P, Magun A (1994). In: IAU symp. Infrared solar physics, vol. 154, pp 125-129

Costa JER, Correia E, Kaufmann P, Magun A, Herrmann R (1995) Sol Phys 159:157

Costa JER, Simões PJA, Giménez de Castro CG (2013), in preparation

Covington AE (1973) Sol Phys 33:439

Cox DP, Tucker WH (1969) Astrophys J 157:1157

Cristiani G, Giménez de Castro CG, Luoni ML, Mandrini CH, Rovira MG, Kaufmann P, Machado M (2007a) Adv Space Res 39:1445

Cristiani G, Giménez de Castro CG, Mandrini CH, Machado ME, Silva IDBE, Kaufmann P, Rovira MG (2008) Astron Astrophys 492:215

Cristiani G, Giménez de Castro CG, Mandrini CH, Machado ME, Rovira MG (2009) Adv Space Res 44:1314

Cristiani GD, Giménez de Castro CG, Simões PJA, Mandrini CH, Kaufmann P (2013)

Cristiani G, Martínez G, Mandrini C, Giménez de Castro C, Rovira M, Kaufmann P, Levato H (2005) J Atmos Sol-Terr Phys 67:1744

Cristiani G, Martinez G, Mandrini CH, Giménez de Castro CG, da Silva CW, Rovira MG, Kaufmann P (2007b) Solar Phys 240:271

Croom DL (1970) Sol Phys 15:414

Crusius-Waetzel AR (1991) Astrophys J 368:390 
De Jager C, Kuijpers J, Correia E, Kaufmann P (1987) Sol Phys 110:317

Doschek GA, Feldman U, Rosenberg FD (1977) Astrophys J 215:329

Doschek GA, Mariska JT, Sakao T (1996) Astrophys J 459:823

Dulk GA (1985) Annu Rev Astron Astrophys 23:169

Emslie AG, Smith DF (1984) Astrophys J 279:882

Fleishman GD, Kontar EP (2010) Astrophys J Lett 709:L127

Fleishman GD, Toptygin IN (2007a) Mon Not R Astron Soc 381:1473

Fleishman GD, Toptygin IN (2007b) Phys Rev E 76:017401

Fletcher L, Hannah IG, Hudson HS, Metcalf TR (2007) Astrophys J 656:1187

Fontenla JM, Balasubramaniam KS, Harder J, (2007) Astrophys J 667:1243

Fürst E (1971) Sol Phys 18:84

Gaizauskas V, Tapping KF (1980) Astrophys J 241:804

Garcia HA (1994) Sol Phys 154:275

Giampapa MS, Africano JL, Klimke A, Parks J, Quigley RJ, Robinson RD, Worden SP (1982) Astrophys J Lett 252:L39

Giménez de Castro CG, Raulin J-P, Makhmutov VS, Kaufmann P, Costa JER (1999) Astron Astrophys 140:373

Giménez de Castro CG, Trottet G, Silva-Valio A, Krucker S, Costa JER, Kaufmann P, Correia E, Levato H (2009) Astron Astrophys 507:433

Guidice DA, Castelli JP (1975) Sol Phys 44:155

Heinzel P, Avrett EH (2011) Solar Phys 277:31

Heitler W (1954) Quantum theory of radiation. Clarendon, Oxford

Herrmann R, Magun A, Costa JER, Correia E, Kaufmann P (1992) Sol Phys 142:157

Hudson HS (1975) Sol Phys 45:69

Hudson HS, Ohki K, (1972) Solar Phys 23:155

Hurford GJ, Krucker S, Lin RP, Schwartz RA, Share GH, Smith DM (2006) Astrophys J Lett 644:L93

Jackson JD (1962) Classical electrodynamics

Jelley JV (1955) Br J Appl Phys 6:227

Kaufmann P, Correia E, Costa JER, Vaz AMZ, Dennis BR (1985) Nature 313:380

Kaufmann P, Giménez de Castro CG, Makhmutov VS, Raulin J, Schwenn R, Levato H, Rovira M (2003) J Geophys Res 108:1280

Kaufmann P, Holman GD, Su Y, Gimenez de Castro CG, Correia E, Fernandes LOT, de Souza RV, Marun A, Pereyra P (2012) Sol Phys 279:465

Kaufmann P, Raulin J-P, Correia E, Costa JER, de Castro CGG, Silva AVR, Levato H, Rovira M, Mandrini C, Fernández-Borda R, Bauer OH (2001) Astrophys J 548:L95

Kaufmann P, Raulin J, Melo AM, Correia E, Costa JER, de Castro CGG, Silva AVR, Yoshimori M, Hudson HS, Gan WQ, Gary DE, Gallagher PT, Levato H, Marun A, Rovira M (2002) Astrophys J 574:1059

Kaufmann P, Raulin J de Castro CGG, Levato H, Gary DE, Costa JER, Marun A, Pereyra P, Silva AVR, Correia E (2004) Astrophys J Lett 603:L121

Kaufmann P, Raulin J-P (2006) Phys Plasmas 13:701

Kaufmann P, Strauss FM, Laporte C, Opher R (1980) Astron Astrophys 87:58

Kaufmann P, Trottet G, Giménez de Castro CG, Raulin J, Krucker S, Shih AY, Levato H (2009) Sol Phys $255: 131$

Kašparová J, Varady M, Heinzel P, Karlický M, Moravec Z (2009) Astron Astrophys 499:923

Kiener J, Gros M, Tatischeff V, Weidenspointner G (2006) Astron Astrophys 445:725

Klein K-L (1987) Astron Astrophys 183:341

Klopf M, Kaufmann P, Raulin J (2010). In: American astronomical society meeting abstracts, vol. 216, 404.31

Kosugi T, Dennis BR, Kai K (1988) Astrophys J 324:1118

Kundu MR (1965) Solar radio astronomy. Interscience Publication, New York

Kuznetsov SN, Kurt VG, Myagkova IN, Yushkov BY, Kudela K (2006) Sol Syst Res 40:104

Leibacher JW, Sakurai T, Schrijver K, van Driel-Gesztely L (2010) Sol Phys 263:1. doi:10.1007/s11207010-9553-0

Lindsey CA, Yee S, Roellig TL, Hills R, Brock D, Duncan W, Watt G, Webster A, Jefferies JT (1990) Astrophys J Lett 353:L53

Lingenfelter RE, Ramaty R (1967) Planet Space Sci 15:1303

Louarn P, Le Queau D, Roux A (1987) Sol Phys 111:201

Loukitcheva MA, Solanki SK, White S (2008) Astrophys Space Sci 313:197 
Lüthi T, Lüdi A, Magun A (2004a) Astron Astrophys 420:361

Lüthi T, Magun A, Miller M (2004b) Astron Astrophys 415:1123

Makhmutov VS, Raulin J, Giménez de Castro CG, Kaufmann P, Correia E (2003) Sol Phys 218:211

McClements KG, Brown JC (1986) Astron Astrophys 165:235

McCray R (1966) Science 154:1320

Melo AM, Kaufmann P, Giménez de Castro CG, Raulin J, Levato H, Marun A, Giuliani JL, Pereyra P (2005) IEEE Trans Antennas Propag 53:1528

Melnikov VF, Costa JER, Simoes PJA (2013) Solar Phys, in preparation

Murphy RJ, Dermer CD, Ramaty R (1987) Astrophys J Suppl Ser 63:721

Nita GM, Gary DE, Lee J (2004) Astrophys J 605:528

Pacholczyk AG (1970) Radio astrophysics. Freeman, San Francisco

Qin Z, Li C, Fu Q, Gao Z (1996) Sol Phys 163:383

Ramaty R, Schwartz RA, Enome S, Nakajima H (1994) Astrophys J 436:941

Raulin J, Kaufmann P, Olivieri R, Correia E, Makhmutov VS, Magun A (1998) Astrophys J Lett 498:L173

Raulin J, Vilmer N, Trottet G, Nitta N, Silva AVR, Kaufmann P, Correia E, Magun A (2000) Astron Astrophys 355:355

Raulin JP, Kaufmann P, Giménez de Castro CG, Pacini A-A, Makhmutov VS, Levato H, Rovira M (2003) Astrophys J 592:580

Raulin JP, Makhmutov VS, Kaufmann P, Pacini AA, Lüthi T, Hudson HS, Gary DE (2004) Sol Phys 223:181

Raymond JC, Holman G, Ciaravella A, Panasyuk A, Ko Y, Kohl J (2007) Astrophys J 659:750

Rybicki GB, Lightman AP (1985). In: Rybicki GB, Lightman AP (ed) Radiative processes in astrophysics

Sakai JI, Nagasugi Y (2007a) Astron Astrophys 474:L33

Sakai JI, Nagasugi Y (2007b) Astron Astrophys 470:1117

Sakai JI, Nagasugi Y, Saito S, Kaufmann P (2006) Astron Astrophys 457:313

Share GH, Murphy RJ, Smith DM, Schwartz RA, Lin RP (2004) Astrophys J Lett 615:L169

Shibasaki K, Enome S, Nakajima H, Nishio M, Takano T, Hanaoka Y, Torii C, Sekiguchi H, Kawashima S, Bushimata T, Shinohara N, Koshiishi H, Shiomi Y, Irimajiri Y, Leka KD, Canfield RC (1994) Publ Astron Soc Jpn 46:L17

Shimabukuro FI (1972) Solar Phys 23:169

Silva ARV, Laganá T, Giménez de Castro CG, Kaufmann P, Costa JER, Levato H, Rovira M (2005) Sol Phys 227:265

Silva AVR, Share GH, Murphy RJ, Costa JER, de Castro CGG, Raulin J, Kaufmann P (2007) Sol Phys 245:311

Slottje C (1978) Nature 275:520

Stähli M, Gary DE, Hurford GJ (1989) Sol Phys 120:351

Staehli M, Magun A, Schanda E (1987) Sol Phys 111:181

Treumann RA (2006) Astron Astrophys Rev 13:229

Trottet G, Krucker S, Lüthi T, Magun A (2008) Astrophys J 678:509

Trottet G, Raulin J, Kaufmann P, Siarkowski M, Klein K, Gary DE (2002) Astron Astrophys 381:694

Trottet G, Raulin J-P, Giménez de Castro G, Lüthi T, Caspi A, Mandrini CH, Luoni ML, Kaufmann P (2011) Sol Phys 273:339

Trottet G, Rolli E, Magun A, Barat C, Kuznetsov A, Sunyaev R, Terekhov O (2000) Astron Astrophys 356:1067

Trottet G, Vilmer N, Barat C, Benz A, Magun A, Kuznetsov A, Sunyaev R, Terekhov O (1998) Astron Astrophys 334:1099

Tsuneta S, Ichimoto K, Katsukawa Y, Nagata S, Otsubo M, Shimizu T, Suematsu Y, Nakagiri M, Noguchi M, Tarbell T, Title A, Shine R, Rosenberg W, Hoffmann C, Jurcevich B, Kushner G, Levay M, Lites B, Elmore D, Matsushita T, Kawaguchi N, Saito H, Mikami I, Hill LD, Owens JK (2008) Sol Phys 249:167

Tsytovich VN, Stenflo L, Wilhelmsson H (1975) Phys Scr 11:251

Turon PJ, Léna PJ (1970) Sol Phys 14:112

Valio A, Kaufmann P, Giménez de Castro CG, (2013) Solar Phys 23

Vernazza JE, Avrett EH, Loeser R (1981) Astrophys J Suppl Ser 45:635

Vilmer N, MacKinnon AL, Trottet G, Barat C (2003) Astron Astrophys 412:865

Vilmer N, Trottet G, Barat C, Schwartz RA, Enome S, Kuznetsov A, Sunyaev R, Terekhov O (1999) Astron Astrophys 342:575

Wang H (2009) Res Astron Astrophys 9:127 
White SM, Benz AO, Christe S, Fárník F, Kundu MR, Mann G, Ning Z, Raulin J-P, Silva Válio AVR, Saint-Hilaire P, Vilmer N, Warmuth A (2011) Space Sci Rev 159:225

Woods TN, Kopp G, Chamberlin PC (2006) J Geophys Res 111:10

Wu CS (1985) Space Sci Rev 41:215

Zheleznyakov VV (1967) Sov Phys JETP 24:381

Zirin H, Wang H (1993) Nature 363:426 